\title{
A New Energy-Efficient Adaptive Clustering Protocol Based on Genetic Algorithm for Improving the Lifetime and the Stable Period of Wireless Sensor Networks
}

\author{
Mohammed Abo-Zahhad ${ }^{1}$, Sabah M. Ahmed ${ }^{1}$, Nabil Sabor ${ }^{1}$ and Shigenobu \\ Sasaki $^{2}$ \\ ${ }^{1}$ Electrical and Electronics Engineering Department, Faculty of Engineering, \\ Assiut University, Assiut, Egypt, (zahhad@yahoo.com, sabahma@yahoo.com, \\ nabil.kolta@eng.au.edu.eg). \\ ${ }^{2}$ Department of Electrical and Electronic Engineering, Niigata University, \\ Niigata-shi,950-2181 Japan, (kojiro@eng.niigata-u.ac.jp)
}

\begin{abstract}
This paper presents a new Genetic Algorithm-based Energy-Efficient adaptive clustering hierarchy Protocol (GAEEP) to efficiently maximize the lifetime and to improve the stable period of Wireless Sensor Networks (WSNs). The new protocol is aimed at prolonging the lifetime of WSNs by finding the optimum number of cluster heads (CHs) and their locations based on minimizing the energy consumption of the sensor nodes using genetic algorithm. The operation of the GAEEP is broken up into rounds, where each round begins with a set-up phase, when the base station finds the optimum number of $\mathrm{CH}$ s and assigns members nodes of each $\mathrm{CH}$, followed by a steady-state phase, when the sensed data are transferred to $\mathrm{CH}$ s and collected in frames; then these frames are transferred to the base station. The performance of the GAEEP is compared with previous protocols using Matlab simulation. Simulation results show that GAEEP protocol improves the network lifetime and stability period over previous protocols in both homogeneous and heterogeneous cases. Moreover, GAEEP protocol increases the reliability of clustering process because it expands the stability period and compresses the instability period.
\end{abstract}

Keywords: Wireless Sensor Networks, Genetic Algorithm, Clustering Protocols, Network Lifetime, Homogeneous and Heterogeneous Networks

\section{Introduction}

Wireless Sensor Networks (WSNs) is widely considered as one of the most important technologies for the twenty-first century. A WSN typically consists of a large number of battery-powered wireless sensor nodes [1]. In most cases it is very difficult and even impossible to change or recharge batteries for the sensor nodes due to the large number of nodes in network and the complexity of environment. Classical approaches like Direct Transmission (DT) and Minimum Transmission Energy (MTE) do not guarantee well balanced distribution of the energy load among sensors of the sensor network. In DT, sensors transmit its sensed data directly to the Base Station (BS); as a result, sensors that are far away from the BS would die first. On the other hand in MTE, sensors that are near the BS would die first because they act as relays for sensors that are far from the BS. This means that the classical approaches will not guarantee good covered of the sensor field [2]. Therefore, designing energy-aware clustering protocols becomes an important factor for extending the lifetime of sensors.

Grouping sensor nodes into clusters has been widely pursued by the research community in order to achieve the network scalability objective and to extend the network 
lifetime. Every cluster would have a leader, often referred to as the Cluster-Head $(\mathrm{CH})$. A $\mathrm{CH}$ may be selected by the sensors in a cluster or by BS or pre-assigned by the network designer. Clustering can also conserve communication bandwidth since it limits the intercluster collisions. Moreover, clustering can also improve data aggregation mechanisms, reduce the workload of each sensor to save energy and thus increase the overall lifetime of the system [3]. Recently, a number of clustering algorithms have been specifically designed for WSNs [4]-[17]. These clustering protocols widely vary depending on the nodes deployment, the network architecture, network and radio models and the characteristics of the $\mathrm{CH}$ nodes.

Clustering algorithm can effectively organize sensor nodes. This clustering problem is considered to be Non-deterministic Polynomial-time hard (NP-hard) problem, and evolutionary algorithms have been applied successfully to a variety of NP-hard problems [18]. So in this paper, a new Genetic Algorithm-based Energy-Efficient adaptive clustering hierarchy Protocol (GAEEP) is developed to efficiently maximize the network lifetime. GAEEP uses Genetic Algorithm (GA) to find the optimum number of CHs and their locations to maximize the network lifetime based on minimizing the energy dissipation of all sensor nodes in communication process.

The rest of the paper is organized as follows. Section 2 is a literature survey about various clustering protocols. The network and radio models that are used in the proposed protocol are adopted in Section 3. Section 4 describes the proposed GAEEP protocol and how the GA is used to find the optimum number of CHs and their locations. In Section 5, the simulation results and discussion are given. Finally, section 6 offers some conclusions.

\section{Related Work}

Many routing protocols based on clustering method for WSNs have appeared in the literature [4-17]. The first low-energy adaptive clustering hierarchy was LEACH, that proposed by Heinzelman et al., [4]. In LEACH protocol, the $\mathrm{CH}$ collects and aggregates sensed data from sensors in its own cluster and passes the aggregated signal to the BS directly. $\mathrm{CH}$ might be located far away from the BS, so it uses most of its energy for transmission and because it is always on, it will die faster than other nodes. In [5], the authors extend LEACH algorithm by making residual energy of node decides whether this node turn into $\mathrm{CH}$ or not in the next round and this improved the performance of LEACH. This algorithm called energy-LEACH.

A new version of LEACH called Two-Level LEACH (TL-LEACH) was presented in [6]. In this protocol, another level in the cluster is added to the respect of LEACH that considers only a one level. This new level reduced the consumption energy for transmitting especially when the $\mathrm{CH}$ located for away from BS. The work described in [7] proposed Vice-CH LEACH (V-LEACH) protocol. In V-LEACH besides having a $\mathrm{CH}$ in the cluster, there is a vice- $\mathrm{CH}$ that takes the role of the $\mathrm{CH}$ and makes the cluster always connected to $\mathrm{BS}$ when the $\mathrm{CH}$ dies. The $\mathrm{LEACH}$ protocol requires the user to specify the desired probability of $\mathrm{CHs}$ for use with the threshold function in determining whether a node becomes a CH or not. However, Genetic Algorithm based LEACH (LEACH-GA) proposed in [8] determines the optimal threshold probability $\left(p_{\text {opt }}\right)$ for cluster formation in WSNs using GA. LEACH-GA improves the $\mathrm{CHs}$ threshold function, but still $\mathrm{CHs}$ are randomly selected and the residual energy of each node is not considered in cluster formation.

A new protocol called Amend LEACH (A-LEACH) was developed in [9], for electing $\mathrm{CHs}$ in a distributed fashion in two-level hierarchical WSNs. Unlike LEACH, A-LEACH is heterogeneous aware, in the sense that election probabilities are weighted by the initial energy of a node relative to that of other nodes in the network. This prolongs the time interval before the death of the first node, which is crucial for many applications. In [10], Enhanced-LEACH (En-LEACH) protocol was adopted to handle $\mathrm{CH}$ failure and to 
account for the non-uniform and dynamic residual energy of the nodes. The Energy Aware Threshold based Efficient Clustering (EATEC) protocol was introduced in [11]. In EATEC, the change of $\mathrm{CH}$ totally depends on the energy variable. The $\mathrm{CH}$ is only changed whenever the current $\mathrm{CH}$ looses down its energy below that of the threshold value.

Energy-LEACH (E-LEACH) algorithm in [12] introduced the concept of the energy threshold and a distance factor in selecting $\mathrm{CH}$. Energy threshold is to determine whether the node can be used as a prerequisite of the $\mathrm{CH}$ node and the distance factor is used to select the path with the smallest data transmission distance. The Improved and Balanced Leach Protocol (IBLEACH) in [13], is an extension of the LEACH, which improves the stable region of the clustering hierarchy and decreases the probability of failure nodes using the characteristic parameters of heterogeneity. In [14] the Kmedoids-LEACH (K$\mathrm{LEACH}$ ) protocol was described to improve the clustering and $\mathrm{CHs}$ selection procedure. For the first round of communication, in setup phase the K-medoids algorithm was used for cluster formation, which ensures uniform clustering.

Author in [15] suggested a heterogeneous-aware protocol to prolong stability period, which is crucial for many applications where the feedback from the sensor network must be reliable. It is called Stable Election Protocol (SEP). SEP is based on weighted election probabilities of each node to become $\mathrm{CH}$ according to the remaining energy in each node. In [16] an Evaluation based clustered Routing Protocol (ERP) was presented to prolong the network lifetime. The idea of ERP is the incorporation of two clustering aspects, namely compactness and separation error criteria, in the fitness function to direct the search into promising solutions. A new Distributed Energy-efficient Unequal clustering routing protocol (DEU) was presented in [17] to balance energy consumption among CHs. DEU combines uneven clustering and multi-hop routing mechanism. Also, DEU protocol employs timer to select the nodes with high energy as CHs to save the energy of nodes in network. CHs consider candidate nodes' optimal hop count, residual energy, inter-cluster and intra-cluster communication cost to select relay nodes.

\section{Network and Radio Models}

In the proposed GAEEP protocol, the following assumptions about network model are fixed:

- The BS is resource-rich device.

- All sensor nodes are stationary after deployment.

- All sensors have GPS or other location determination devices

- Nodes located close to each other have correlated data.

- The communication channel is symmetric (i.e., the energy required to transmit a message from sensor node $s_{1}$ to sensor node $s_{2}$ is the same as energy required to transmit a message from node $s_{2}$ to node $s_{1}$ for a given signal to noise ratio).

For fair comparison with previous protocols $[4,8,9]$ and [15-17], we assume the simple model shown in Figure (1) for the radio hardware energy dissipation where the transmitter dissipates energy to run the radio electronics and the power amplifier, and the receiver dissipates energy to run the radio electronics. For the experiments described here, both the free space ( $d^{2}$ power loss) and the multipath fading ( $d^{4}$ power loss) channel models were used, depending on the distance between the transmitter and receiver. Thus, to transmit a $k$-bit message a distance $d$, the radio expends:

$$
E_{T x}(k, d)=E_{T x-\text { elec }}(k)+E_{T x-\text { amp }}(k, d)= \begin{cases}k E_{\text {elec }}+E_{f s} k d^{2}, & \text { if } d<d_{o} \\ k E_{\text {elec }}+E_{m p} k d^{4}, & \text { if } d \geq d_{o}\end{cases}
$$

and to receive this message, the radio expends: 


$$
E_{R x}(k)=E_{R x-\text { elec }}(k)=k E_{\text {elec }}
$$

where $d_{o}=\sqrt{E_{f s} / E_{m p}}$ denotes the threshold distance and the electronics energy $\left(E_{\text {elec }}\right)$ depends on factors such as the digital coding, modulation, filtering, and spreading of the signal, whereas the amplifier energy $E_{f s} d^{2}$ or $E_{m p} d^{4}$ depends on the distance to the receiver and the acceptable bit-error rate. For illustration, the communication energy parameters are set as $E_{\text {elec }}=50 \mathrm{~nJ} / \mathrm{bit}, E_{f s}=10 \mathrm{pJ} / \mathrm{bit} / \mathrm{m}^{2}$, and $E_{m p}=0.0013 \mathrm{pJ} / \mathrm{bit} / \mathrm{m}^{4}$. The energy for data aggregation is set as $E_{D A}=5 \mathrm{~nJ} / \mathrm{bit} / \mathrm{signal}$.

Assume that there are $N$ nodes randomly distributed in an $M \times M$ region. If there are $L$ clusters and each cluster has $m_{i}$ member nodes (where $i=1,2, \ldots, L$ ). The $\mathrm{CH}$ dissipates energy in receiving signals from its member nodes, aggregating the received signals and transmitting the aggregated signal to the BS. Therefore, the energy dissipated in the $i^{\text {th }} \mathrm{CH}$ node during a single frame is given by Equation (3), where $k$ is the number of bits in each data message, $d_{i-t o B S}$ is the distance between the $i^{\text {th }} \mathrm{CH}$ node and the BS, and perfect data aggregation is assumed.

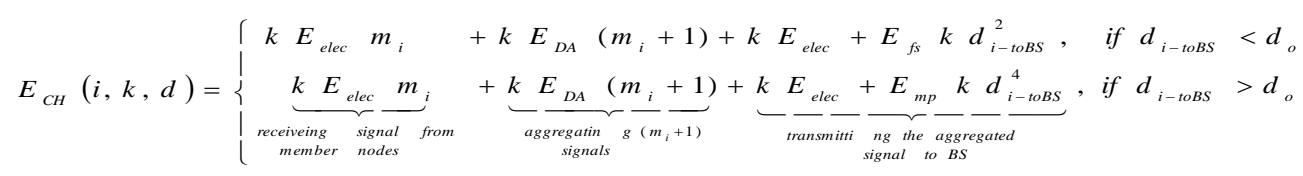

Each member node only needs to transmit its sensed data to the $i^{\text {th }} \mathrm{CH}$ once during a frame. Thus, the energy dissipated in each member node is given by Equation (4); where $d_{l-t o C H}$ is the distance from $l^{\text {th }}$ member node to its $\mathrm{CH}$.

$$
\begin{aligned}
& E_{m e m-i}(l, k, d)=\left\{\begin{array}{llll}
k & E_{e l e c}+E_{f s} & k & d_{l-t o C H}^{2}, \text { if } d_{l-t o C H},<d_{o} \\
k & E_{e l e c}+E_{m p} & k & d_{l-t o C H}^{4},
\end{array}, \text { if } d_{l-t o C H},>d_{o}\right.
\end{aligned}
$$

Figure 1. Radio Energy Dissipation Model

\section{Genetic Algorithm-based Energy-Efficient Protocol}

Grouping sensor nodes into clusters has been widely pursued by the research community in order to achieve the network scalability and to maximize the network lifetime. Every cluster would have a leader, often referred to as the $\mathrm{CH}$ as shown in Figure (2). The clustering problem is considered to be NP-hard problem, and evolutionary algorithms have been applied successfully to a variety of NP-hard problems [18]. For a given network topology, it is difficult to find the optimal number of the $\mathrm{CHs}$ and their locations. Consider a 100 node example; to perform an exhausted search of all possible solutions requires $c_{100}^{1}+c_{100}^{2}+\cdots+c_{100}^{100}=2^{100}-1$ different combinations which is far too large to be handled by existing computer resources.

A GA is an efficient search algorithm that mimics the adaptive evolution process of natural systems. It has been successfully applied to many NP-hard problems such as multi-processor task scheduling, optimization, and traveling sales man problems [20][21]. We propose to apply a GA to the problem of finding optimum number of $\mathrm{CHs}$ based on minimizing the communication consumption energy of all sensor nodes to efficiently maximize the network lifetime and to improve the stability period. The operation of the GAEEP protocol is broken up into rounds, where each round begins with a set-up phase, when the $\mathrm{BS}$ finds the locations of $\mathrm{CHs}$ and assigns members nodes of each $\mathrm{CH}$, followed 
by a steady-state phase, when the sensed data are transferred to $\mathrm{CHs}$ and collect in frames; then these frames are transferred to the BS as shown in Figure (3).

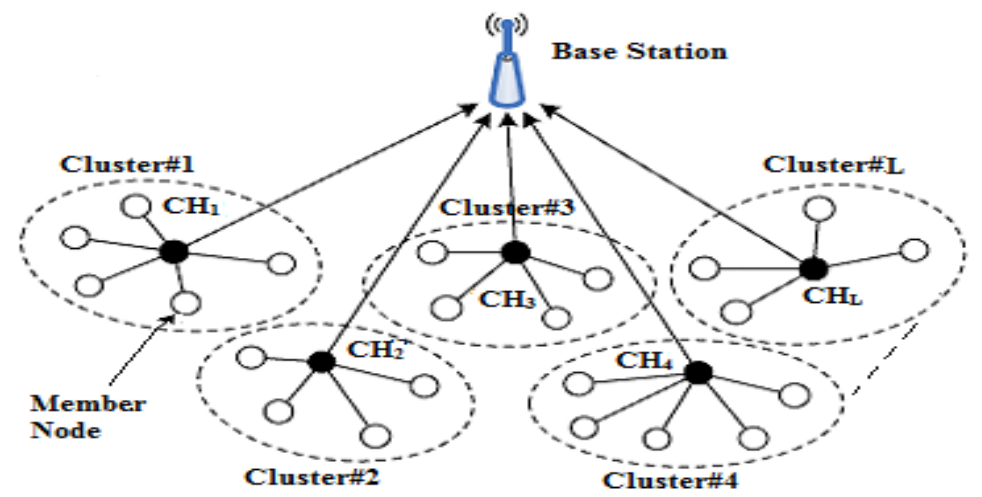

Figure 2. A Hierarchical Clustering

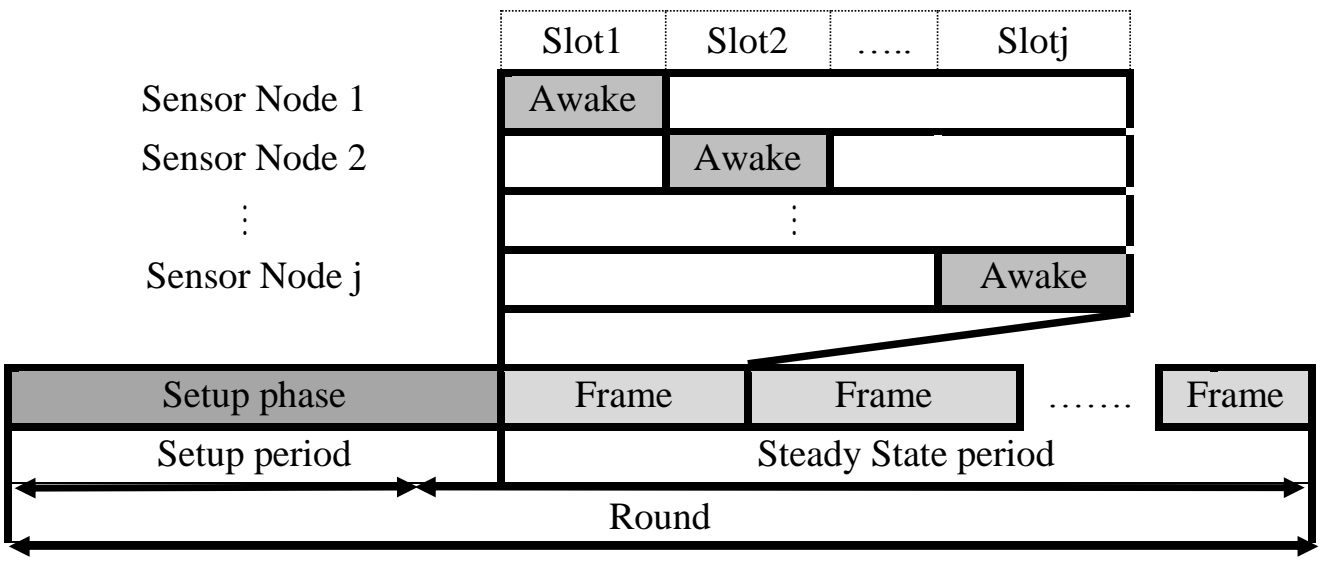

Figure 3. One Round in Operation of the Proposed Protocol

\subsection{Set-up Phase}

In set-up phase, the optimum number of clusters is found and clusters are created as shown in Figure (4). In first round, the BS sends a short message to wake up and to request the IDs, positions and energy levels of all sensor nodes in the sensor field. Based on the feedback information from sensor nodes, the BS uses GA to find the optimum number of $\mathrm{CHs}$ and their locations based on minimization of the dissipated energy on communication process as shown by shaded block in Figure (4). This shaded block is illustrated in Figure (5). Also, the BS assigns the members nodes of each $\mathrm{CH}$. If a sensor node is near to $\mathrm{BS}$ than any $\mathrm{CH}$, this node will communicate directly to $\mathrm{BS}$. Once $\mathrm{CHs}$ are selected and members of $\mathrm{CH}$ are assigned, the BS sends a short message to inform each $\mathrm{CH}$ by IDs of its member nodes then send a short message contains CH's ID that each member node associated with it and logic ' 0 ' to inform each member node where will be join.

Based on a short message received from the sink, each $\mathrm{CH}$ creates the TDMA schedule by assigning slots to its member nodes and informs these nodes by this schedule. The TDMA schedule is used to avoid intra-cluster collisions and reduce energy consumption between data messages in the cluster and enables each member of the radio equipment off when not in use. Also, to reduce inter-cluster interference every $\mathrm{CH}$ selects a unique CDMA code and informs all member nodes within the cluster to transmit their data using this spreading code. The details of the proposed GAEEP protocol and how GA is used to find the $\mathrm{CHs}$ as follows: 


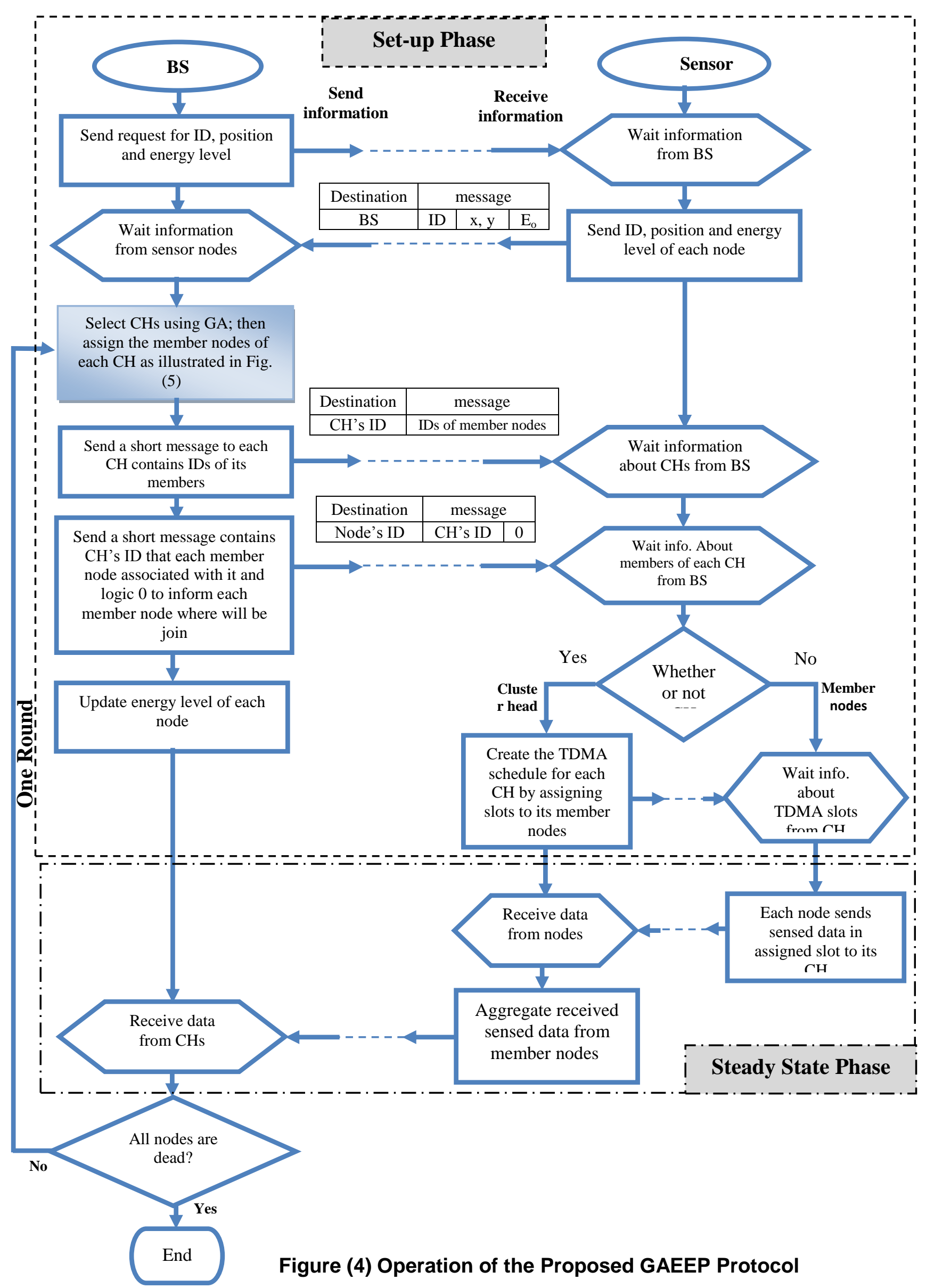




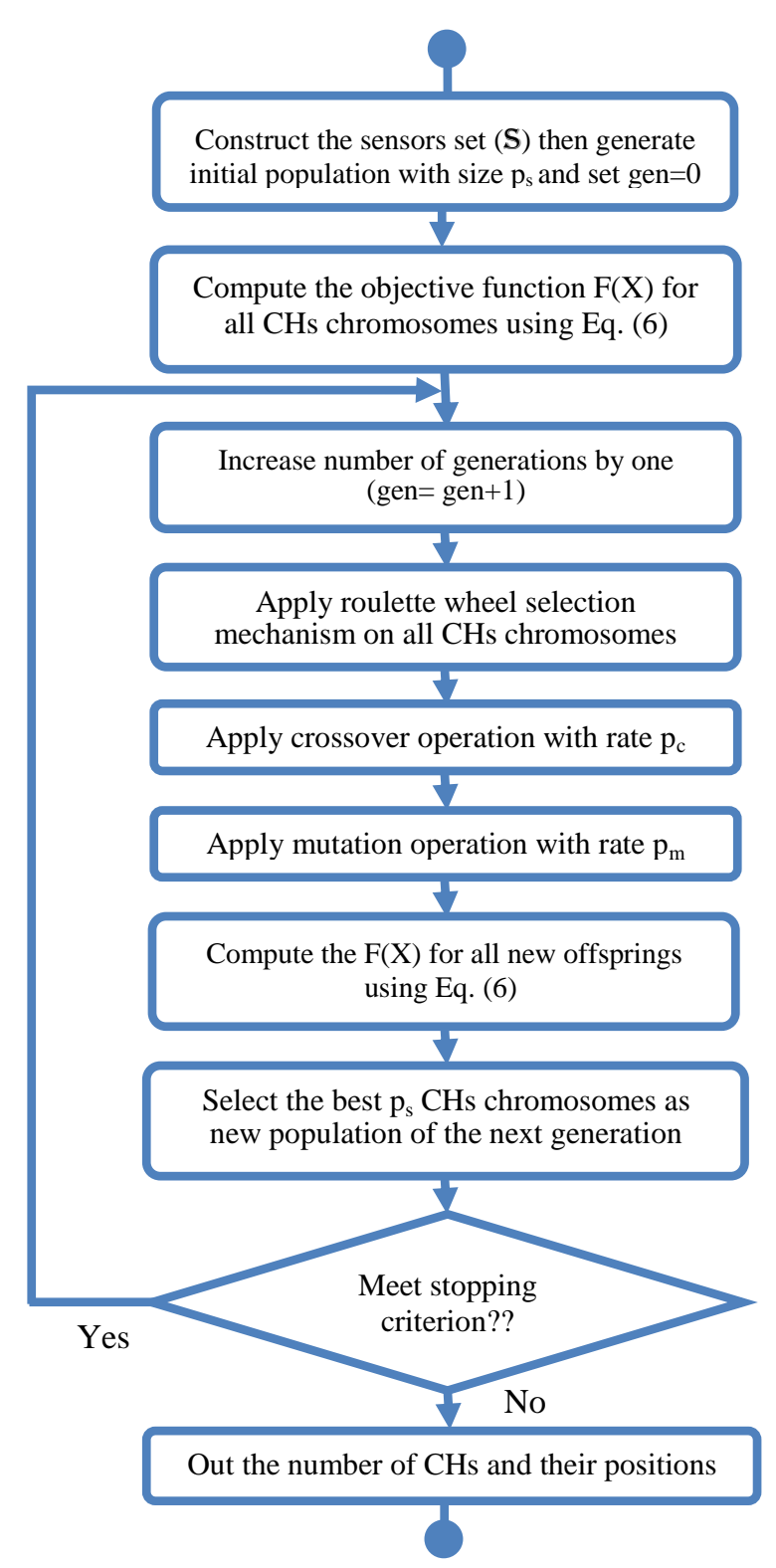

\section{Figure 5. Flow Chart of Cluster-Head Selection Algorithm in the Proposed Protocol}

\section{1) Problem Representation}

Finding appropriate $\mathrm{CHs}$ is critically important to minimizing the consumption energy. In the algorithm shown in Figure (5), BS constructs the sensors set, $\mathbb{S}=\left\{S_{1}, S_{2}, \ldots, S_{N_{\mathbb{S}}}\right\}$, by selecting the sensors nodes that have residual energy equal or larger than the average energy of all live nodes as follows:

$\forall i=\left\{1,2, \cdots, N_{\text {live }}\right\}: \mathrm{S}_{i} \in \mathbb{S}$, if $E_{R S_{i}} \geq E_{\text {Avg }}$

where, $\mathbb{S} \subset\left\{\mathrm{S}_{1}, \mathrm{~S}_{2}, \ldots, \mathrm{S}_{N_{\text {live }}}\right\}, N_{\mathbb{S}} \leq N_{\text {live }}$ is number of the sensors nodes in set $\mathbb{S}, N_{\text {live }}$ is number of the live nodes in the sensor field, $E_{R S_{i}}$ is the residual energy of $i^{\text {th }}$ sensor and $E_{A v g}$ is the average of residual energy of all live nodes in the sensor field.

Then BS forms chromosome using a binary stream $\mathrm{X}$ with length $N_{\mathbb{S}}$, where $N_{\mathbb{S}}$ is number of sensors nodes in set $\mathbb{S}$, in which each bit corresponds to one sensor node in the sensors set $\mathbb{S}$. A " 1 " means that corresponding node is a $\mathrm{CH}$; otherwise, it is a regular 
node. For example shown in Table 1 , sensor set contains 10 sensors nodes, $\mathbb{S}=$ $\left\{\mathrm{S}_{1}, \mathrm{~S}_{2}, \ldots, \mathrm{S}_{10}\right\}$, where nodes $\mathrm{S}_{1}, \mathrm{~S}_{2}, \mathrm{~S}_{7}, \mathrm{~S}_{9}$ and $\mathrm{S}_{10}$ are $\mathrm{CHs}$ and the remaining nodes are regular nodes. The initial population consists of randomly generated $\mathrm{CHs}$ chromosomes with size $p_{s}$ and each $\mathrm{CHs}$ chromosome has length $N_{\mathbb{S}}$.

Table 1. Binary Representation of Sensor Nodes

\begin{tabular}{|c|l|l|l|l|l|l|l|l|l|l|}
\hline Sensors set (S) & $\mathbf{S}_{\mathbf{1}}$ & $\mathbf{S}_{\mathbf{2}}$ & $\mathbf{S}_{\mathbf{3}}$ & $\mathbf{S}_{\mathbf{4}}$ & $\mathbf{S}_{\mathbf{5}}$ & $\mathbf{S}_{\mathbf{6}}$ & $\mathbf{S}_{\mathbf{7}}$ & $\mathbf{S}_{\mathbf{8}}$ & $\mathbf{S}_{\mathbf{9}}$ & $\mathbf{S}_{\mathbf{1 0}}$ \\
\hline CHs chromosome (X) & 1 & 1 & 0 & 0 & 0 & 0 & 1 & 0 & 1 & 1 \\
\hline
\end{tabular}

\section{2) Objective Function Evaluation}

The dissipation energy in communication process is the main factors we need to minimize. In addition, the number of $\mathrm{CHs}$ can factor into the objective function. Fewer $\mathrm{CHs}$ result in greater energy efficiency and higher $\mathrm{CHs}$ consume more energy as $\mathrm{CHs}$ drain more power than non-cluster heads. Therefore, GA is used to determine optimal number of $\mathrm{CHs}$ and their locations by minimizing the following objective function $\mathrm{F}(\mathrm{X})$ :

$$
F(X)=w\left(\frac{E_{\text {disp }}}{E_{\text {live }}}\right)+(1-w)\left(\frac{L}{N_{\text {live }}}\right)
$$

where $E_{\text {live }}$ is the total energy for all live nodes in sensor field. It is described by:

$$
E_{\text {live }}=\sum_{j=1}^{N_{\text {live }}} E_{o}(j)=N_{\text {live }} E_{o}
$$

and $E_{\text {disp }}$ is the total dissipated energy of all live nodes in the sensor field. It is given by:

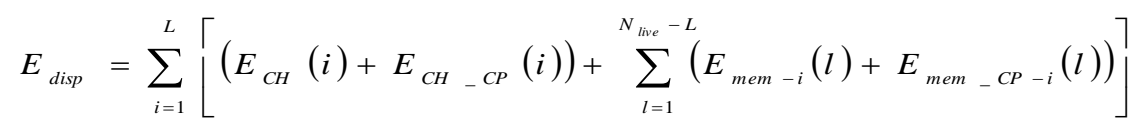

where $L$ is the number of CHs; $E_{o}$ is the initial energy of sensor nodes, $N_{\text {live }}$ is the number of nodes alive in the sensor field (i.e., $N_{\text {live }}=N$ if all nodes are live), and $w$ is a pre-defined weighting factor $(0 \leq w \leq 1) . E_{C H_{-} C P}$ and $E_{m e m_{-} C P-i}$ are the dissipated energy in control packets for $\mathrm{CH}$ and member node respectively and are given by:

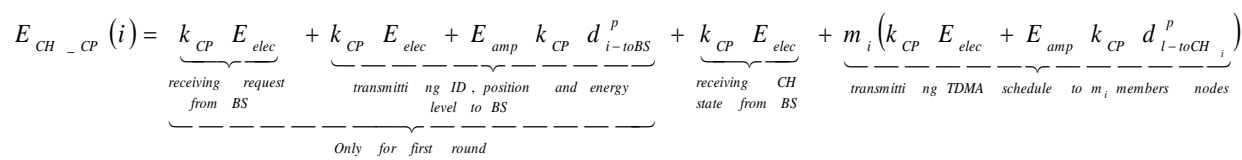

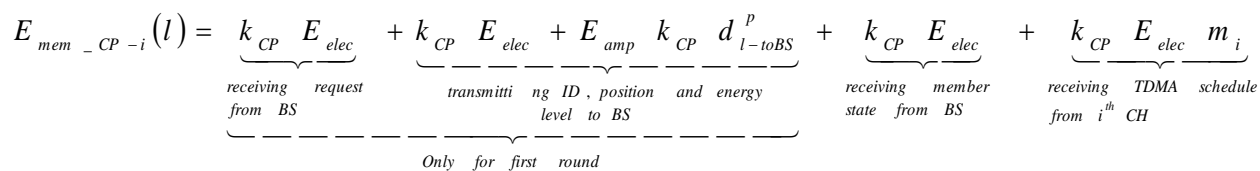

where $k_{C P}$ is the number of bits in each control packet, $d_{i-\text { toBS }}$ is the distance between the $i^{\text {th }}$ $\mathrm{CH}$ node and the BS, $d_{\text {l-toBS }}$ is the distance from the $l^{\text {th }}$ member node to the BS and $d_{l-t o C H}$ is the distance between $l^{\text {th }}$ member node and its $\mathrm{CH}$. The path loss $(p)$ equals 4 if distance larger than $d o$ and equals 2 otherwise 


\section{3) GA Operators}

Crossover and mutation provide exploration, compared with the exploitation provided by selection. The effectiveness of GA depends on the trade-off between exploitation and exploration [20]-[21].

\section{A) Selection}

The selection process determines which of the $\mathrm{CHs}$ chromosomes from the current population will mate (crossover) to create new chromosomes. These new chromosomes join the existing population. This combined population will be the basis for the next selection. Roulette wheel selection is used in the proposed algorithm. Its basic idea is to determine the selection probability for each $\mathrm{CHs}$ chromosome in proportion with the fitness value $(1 / F(X))$. The $\mathrm{CHs}$ chromosomes with higher fitness values are more likely to be selected as the chromosomes of population in the next generation.

\section{B) Crossover}

The outcome of crossover heavily depends on the selection of chromosomes made from the population. Crossover is a binary genetic operator acting on two parents. The single-point crossover is used here, whereby a point is chosen at random based on crossover rate $p_{c}$, and two parent $\mathrm{CHs}$ chromosomes are selected randomly to exchange information after that point to produce two children. This process is repeated to produce $N_{c}$ children. For example consider two parents $\mathrm{CHs}$ chromosomes; each one consists from 10 sensor nodes. Nodes $S_{1}, S_{2}, S_{7}, S_{9}$ and $S_{10}$ are $\mathrm{CHs}$ in the first parent, but the CHs in the second parent are $S_{3}, S_{4}, S_{6}$ and $S_{7}$ as shown in Table 2. If the crossover point is chosen at sensor node $S_{6}$, the two parents exchange sensor nodes after $S_{6}$. Therefore, two offspring are generated as shown in Table 2.

Table 2. A Single-Point Crossover Example

\begin{tabular}{|c|ccccccccccccccccccccccc|}
\hline & \multicolumn{1}{|c|}{ First } & \multicolumn{1}{|c|}{ Second } \\
\cline { 2 - 3 } & $\mathrm{S}_{1}$ & $\mathrm{~S}_{2}$ & $\mathrm{~S}_{3}$ & $\mathrm{~S}_{4}$ & $\mathrm{~S}_{5}$ & $\mathrm{~S}_{6}$ & $\mathrm{~S}_{7}$ & $\mathrm{~S}_{8}$ & $\mathrm{~S}_{9} \mathrm{~S}_{10}$ & $\mathrm{~S}_{1}$ & $\mathrm{~S}_{2}$ & $\mathrm{~S}_{3}$ & $\mathrm{~S}_{4}$ & $\mathrm{~S}_{5}$ & $\mathrm{~S}_{6}$ & $\mathrm{~S}_{7}$ & $\mathrm{~S}_{8}$ & $\mathrm{~S}_{9}$ & $\mathrm{~S}_{10}$ \\
\hline Parent & 1 & 1 & 0 & 0 & 0 & 0 & 1 & 0 & 1 & 1 & 0 & 0 & 1 & 1 & 0 & 1 & 1 & 0 & 0 & 0 \\
\hline Offspring & 1 & 1 & 0 & 0 & 0 & 0 & 1 & 0 & 0 & 0 & 0 & 0 & 1 & 1 & 0 & 1 & 1 & 0 & 1 & 1 \\
\hline
\end{tabular}

\section{C) Mutation}

The mutation operator is applied to each gene of $\mathrm{CHs}$ chromosome with a probability of mutation $p_{m}$. The probability of mutation determines where the bits will mutate, if $p_{m}$ is 0.001 , each gene in the chromosome has 0.1 percent chance of being mutated. Mutation, unlike crossover, is a unary genetic operator that affects only a single chromosome. A $\mathrm{CHs}$ chromosome selected for mutation will have a randomly selected gene changed from 0 to 1 , or vice versa. Table 3 shows the effect of mutation on the two offspring created as a result of crossover. Based on the mutation probability, the node $S_{5}$ in the first offspring is chosen to mutate from 0 to 1 and becomes $\mathrm{CH}$; however, due to very low mutation probability, there is no mutation in offspring 2.

Table 3. An Example of Mutation

\begin{tabular}{|c|c|c|c|c|c|c|c|c|c|c|c|c|c|c|c|c|c|c|c|}
\hline & \multicolumn{9}{|c|}{ Offspring1 } & \multicolumn{10}{|c|}{ Offspring2 } \\
\hline & $S_{1}$ & $S_{2}$ & $\mathrm{~S}_{3}$ & $\mathrm{~S}_{4}$ & $\mathrm{~S}_{5}$ & $\mathrm{~S}_{6}$ & $\mathrm{~S}_{7}$ & $S_{8}$ & $S_{9} S_{10}$ & & $\mathrm{~S}_{2}$ & $\mathrm{~S}_{3}$ & $\mathrm{~S}_{4}$ & $\mathrm{~S}_{5}$ & $S_{6}$ & $\mathrm{~S}_{7}$ & $S_{8} S$ & ${ }_{9} \mathrm{~S}$ & \\
\hline Original & 1 & 1 & 0 & 0 & 0 & 0 & 1 & 0 & 00 & 0 & 0 & 1 & 1 & 0 & 1 & 1 & 0 & 1 & 1 \\
\hline Mutated & 1 & 1 & 0 & 0 & 1 & 0 & 1 & 0 & 00 & 0 & 0 & 1 & 1 & 0 & 1 & & 0 & 1 & 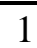 \\
\hline
\end{tabular}




\section{4) Selection of the Best Solutions}

Better chromosomes are always survived. In this step, the initial population pool (parents) and offsprings chromosomes that generated in previous step are sorted in ascending order based on the objective function values. Then the first $p_{s} \mathrm{CHs}$ chromosomes with minimum objective function values are selected to form the population pool for next generation.

\section{5) Stopping Criterion}

The stopping criterion achieves when the objective function does not change for certain number of generations or when the number of generations exceeds the specified maximum generations (Maxgen).

\subsection{Steady State Phase}

During the steady phase, the sensor nodes are wake up and start sensing data. Then each node sends the sensed data to its $\mathrm{CHs}$ according to the TDMA schedule as shown in Figure 4. The $\mathrm{CH}$ node must keep its receiver on to receive all the data from the nodes in the cluster. When all the data have been received, the $\mathrm{CH}$ node performs signal processing functions to aggregate the data into a single signal. This aggregated signal is sent to the BS. After a certain time, which is determined a priori, the network again goes back into the setup phase and new CHs are determined using GA.

The steps of the GAEEP protocol can be summarized as follows:

Step 1: Based the feedback information to BS, the BS initializes the network by defining the parameters of the energy model, $N, E_{o}, k$, sink location and sensor field size.

Step 2: Set the parameters of the GA such as $p_{s}, p_{c}, p_{m}$ and Maxgen; then set rounds counter (r) to zero.

Step 3: BS constructs the sensors set $\mathbb{S}$ by selecting sensors nodes that have residual energy equal or larger than the average energy of all live sensor nodes in the sensor field.

Step 4: Apply GA by setting randomly $p_{s}$ initial $\left(N_{\mathbb{S}}\right)$ bits binary chromosomes and set the counter of generations ( $\mathrm{gen}$ ) to zero.

Step 5: Calculate the objective function $F(X)$ for all $\mathrm{CHs}$ chromosomes using Equation (5).

Step 6: Increase the counter of generations by one $($ gen=gen +1$)$.

Step 7: Select the best CHs chromosomes based on the fitness value $(1 / F(X))$ using roulette wheel selection.

Step 8: From each pair of CHs parents, take $N_{c}$ children by crossover operation based on the crossover rate $p_{c}$.

Step 9: Apply the mutation to all genes of each child generated from step 7 based on probability of mutation $p_{m}$.

Step 10: Calculate the objective function $F(X)$ for new $\mathrm{CHs}$ chromosomes using Equation (5).

Step 11: Update the energy level of all sensor nodes based on the energy model.

Step 12: Select the best $p_{s}$ chromosomes from parents and children to be population pool for the next generation.

Step 13: Has stopping criterion met? If yes, then go to step 14. Otherwise, return to step 6 and continue through step 17.

Step 14: Display the number of $\mathrm{CHs}$ and their locations.

Step 15: Assign the members nodes of each $\mathrm{CH}$. 
Step 16: Steady state phase, when the sensed data transfers to $\mathrm{CHs}$ and collects in frames; then these frames transfers to the BS.

Step 17: Has the energy of all nodes equal or less than zeros? If yes, then stop. Otherwise, increase number of rounds by one and return to step 3 and continue through step 17.

\section{Simulation and Results}

In this section, we perform simulations using Matlab to analyze and evaluate the performance of the proposed Protocol.

\subsection{Performance Measures}

There are many metrics to evaluate the performance of the clustering protocols [2], [21]. These measures are used in this paper to evaluate the performance of GAEEP protocol:

$>$ Network lifetime: The time interval from the start of operation (of the sensor network) until the death of the last alive sensor.

$>$ Stability Period: The time interval from the start of network operation until the death of the first sensor.

$>$ Instability Period: The time interval from the death of the first sensor until the death of the last sensor.

$>$ First Dead Node (FDN): Number of rounds after which the first sensor died. This parameter has direct relation with stability period parameter. That is to say, the bigger FDN is, the longer stability period of network becomes.

$>$ Half Dead Node (HDN): Number of rounds after which half number of sensor nodes are dead.

$>$ Last Dead Node (LDN): Number of rounds after which all sensor nodes are dead.

$>$ Number of Cluster Heads per Round: This instantaneous measure reflects the number of nodes which would send directly to the sink information aggregated from their members.

$>$ Number of Alive Nodes per Round: This instantaneous measure reflects the total number of nodes that have not yet expended all of their energy.

$>$ Throughput: It measures the total rate of data sent over the network including the rate of data sent from $\mathrm{CHs}$ to the sink as well as the rate of data sent from the nodes to their CHs.

Clearly, the larger the stability period and the smaller the instability period are, the better the reliability of the clustering process of the sensor network is.

\subsection{Experiments}

In this subsection several experiments are performed using Matlab to validate the proposed GAEEP protocol and to compare its performance with other protocols. For fair in comparison, the energy consumption due to communication in the following experiments is calculated using the first order energy model that described in Section 3. To eliminate the experimental error caused by randomness, each experiment was run for 5 different networks and the average was taken as the final result.

\subsubsection{Experiment 1}

To compare the proposed protocol with LEACH protocol $[4,19]$, the simulation consists of 100 homogeneous nodes with initial energy of $0.5 \mathrm{~J}$, scattered randomly within a $100 \times 100 \mathrm{~m}^{2}$ sensor field as shown in Figure 6 . The BS was positioned at point $(50,300)$ $\mathrm{m}$ and the packets sent were 2000 bit plus 50 bit control packets. The GA parameters are 
set based on study described in [22] as $p_{s}=50, p_{m}=0.11, p_{c}=0.7$, weighting factor $w$ is 0.95 and maximum number of generation (Maxgen) is 150 .

The homogeneous network after clustering using GAEEP protocol is shown in Figure 7. Figures 8 and 9 show the network lifetime and the average residual energy for all nodes per round respectively for the proposed protocol and LEACH protocol. From these figures, it is noticed that the first died after 364 rounds and all nodes died after 739 rounds in LEACH. However in GAEEP protocol, first node died after 1017.7 rounds and all nodes died after 1175 rounds. It is clearly that the proposed protocol extends the stability period and shrinks the instability period as compared to the LEACH by 653.7 and 217.7 rounds respectively. This means that the proposed protocol increases the reliability of the clustering process in WSN. Moreover, the proposed protocol prolongs the network lifetime more than the LEACH protocol by $58.9986 \%$. This due to that the GAEEP protocol always selects the $\mathrm{CHs}$ from nodes that have energy higher than the average energy of all live nodes. The residual energy of sensors nodes in proposed protocol decreases more slowly than that in LEACH. After 739 rounds the residual energy of all nodes in LEACH dissipates; but the nodes in GAEEP dissipate only $70 \%$ of its initial energy after 739 rounds. This means that the proposed GAEEP protocol improves the network lifetime and saves the residual energy of the sensors nodes.

The total number of packets that send to CHs and BS in GAEEP and LEACH protocols is $1.083 \times 10^{5}$ packets and $5.182 \times 10^{4}$ packets respectively as shown in Figure 10. Figure 11 shows the number of $\mathrm{CHs}$ that selected in each round using $\mathrm{LEACH}$ and GAEEP protocols. From this figure, it is noticed that the number of selected $\mathrm{CHs}$ in each round using LEACH protocol changes from 0 to $11 \mathrm{CHs}$, although the desired number of $\mathrm{CHs}$ is 5. However in GAEEP protocol, number of $\mathrm{CHs}$ is approximately uniform and changes from 1 to $7 \mathrm{CHs}$.

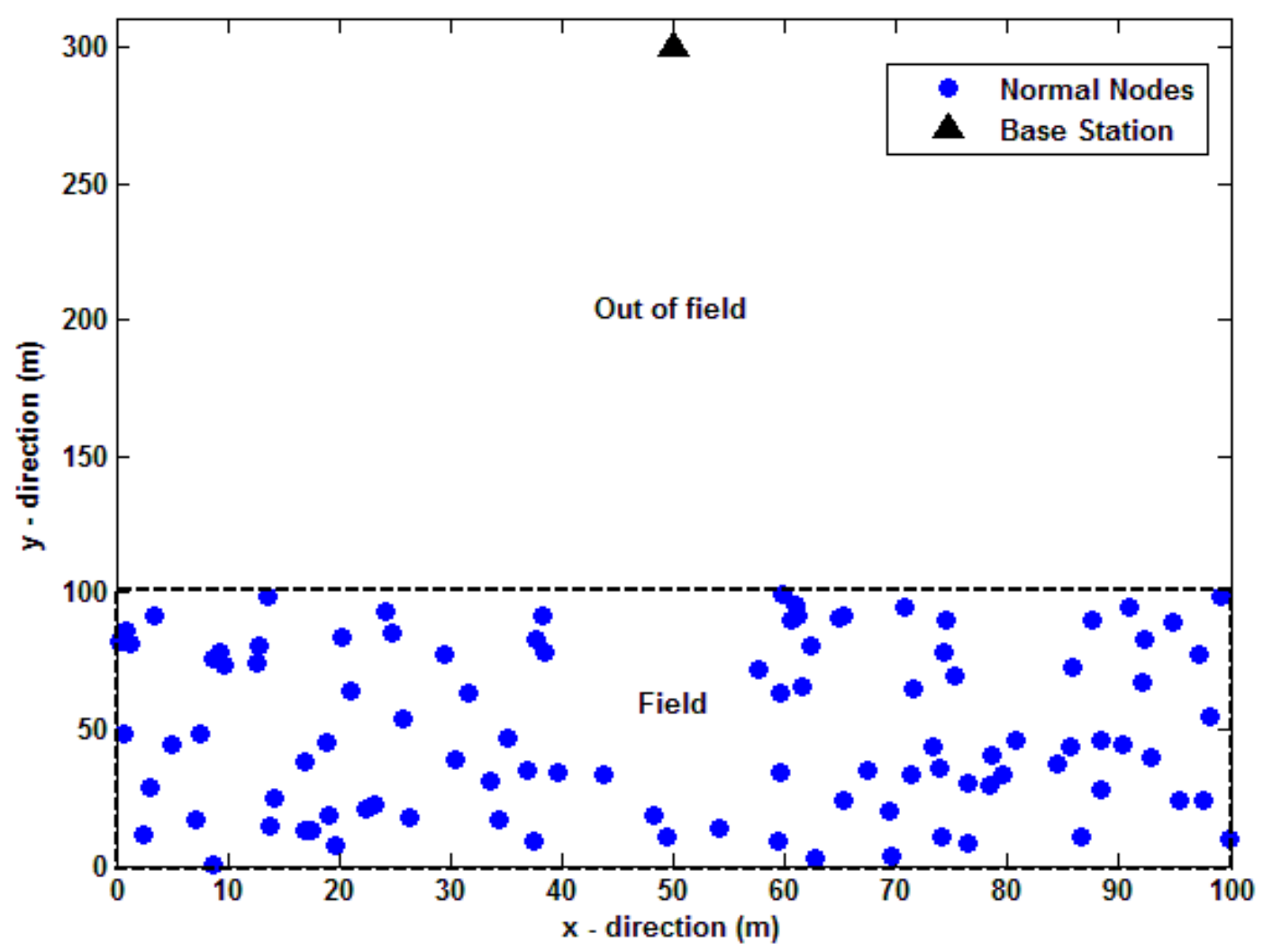

Figure 6. Homogeneous Network before Clustering (Experiment 1) 


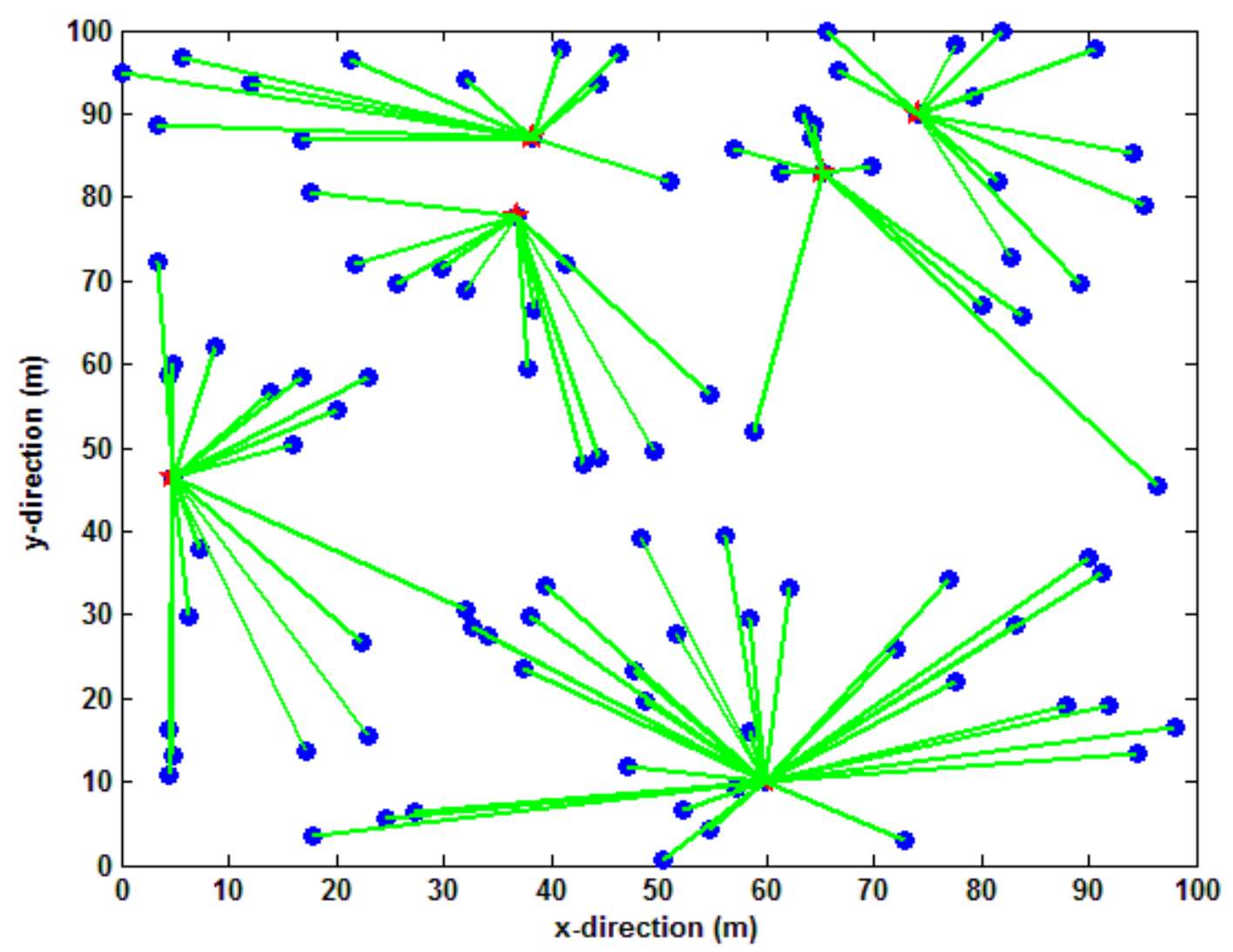

Figure 7. Sensors Field of Homogeneous Network after Clustering (Experiment 1)

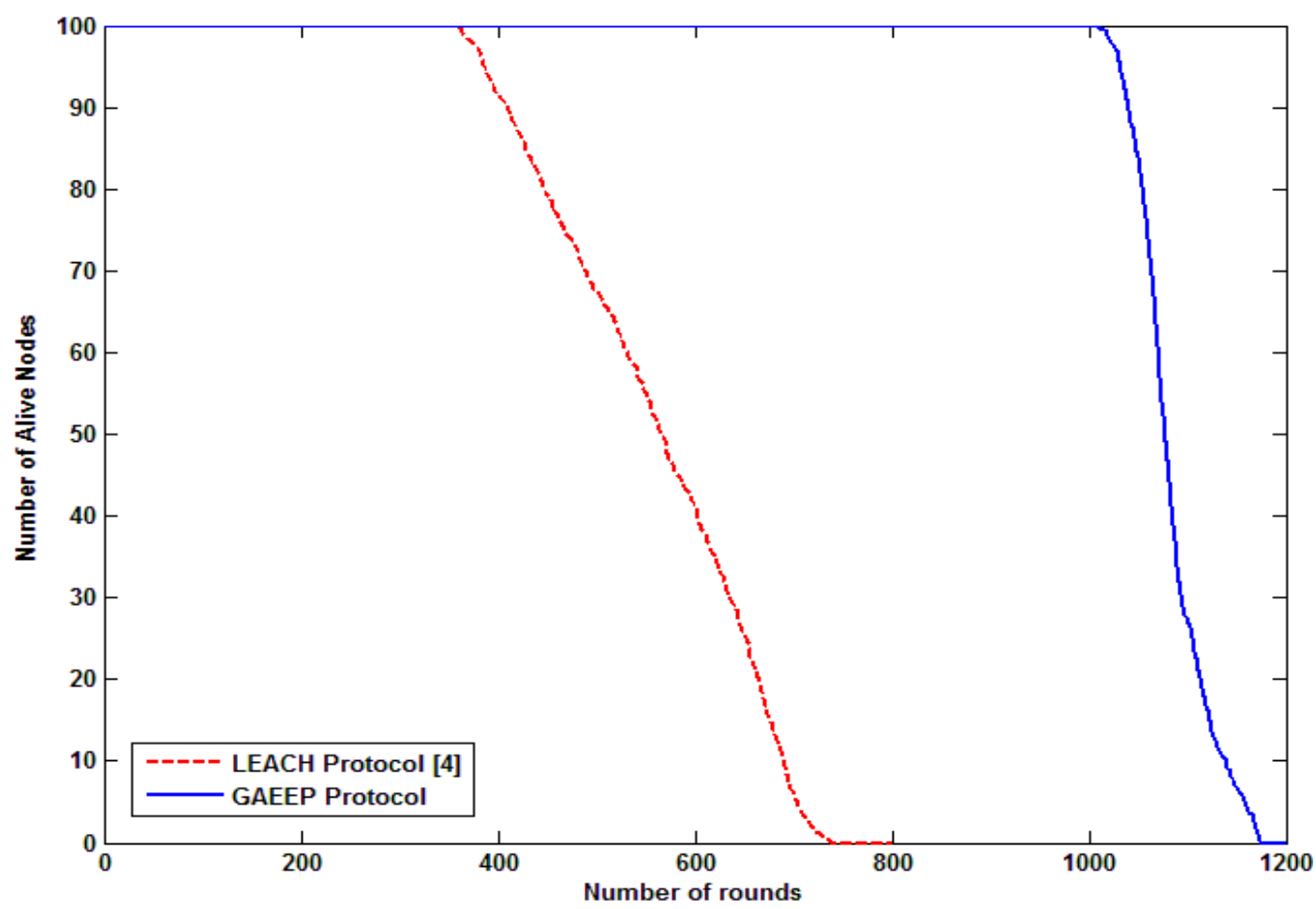

Figure 8. Lifetime of Homogeneous Network (Experiment 1) 


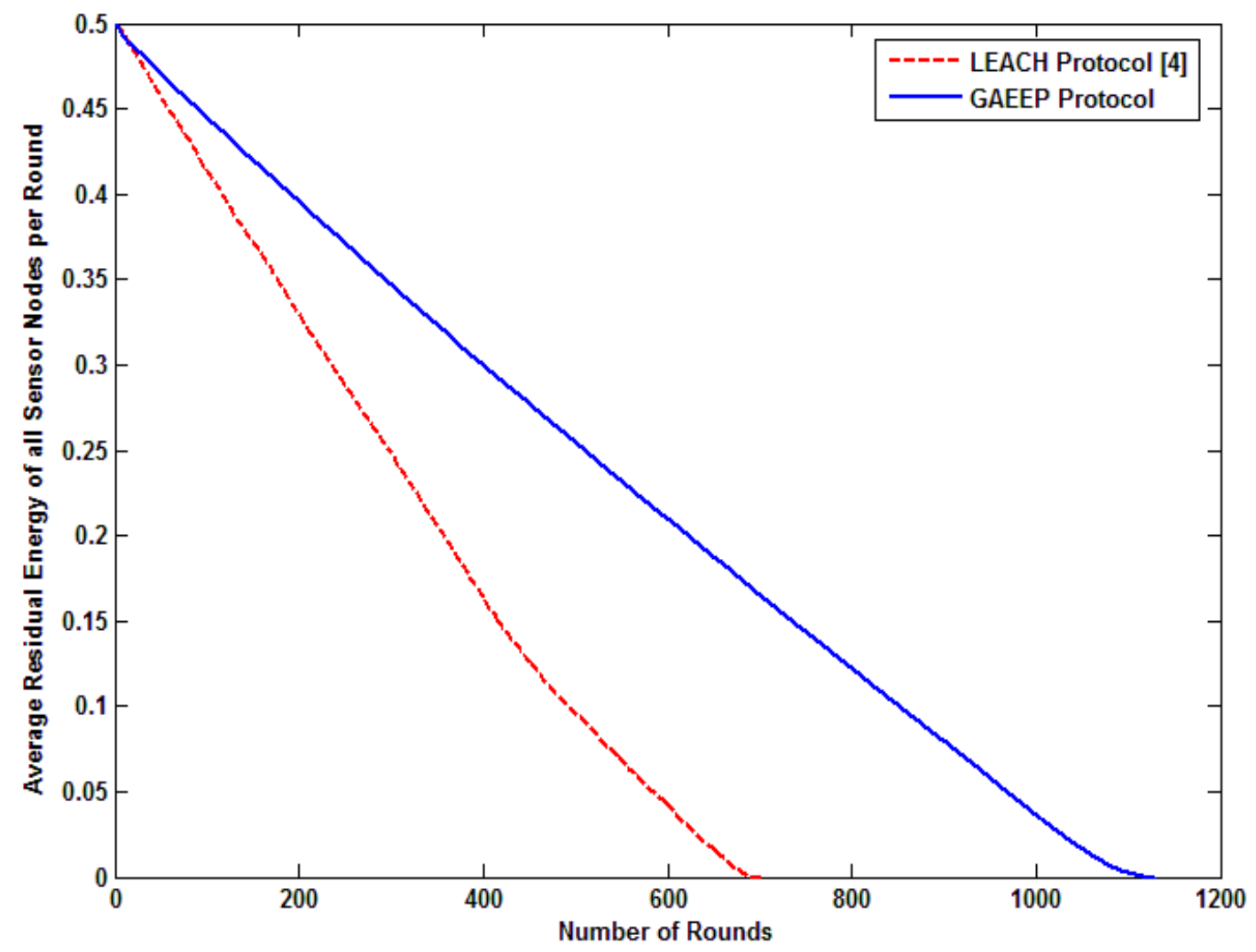

Figure 9. Averaged Residual Energy of all Nodes per Round (Experiment 1)

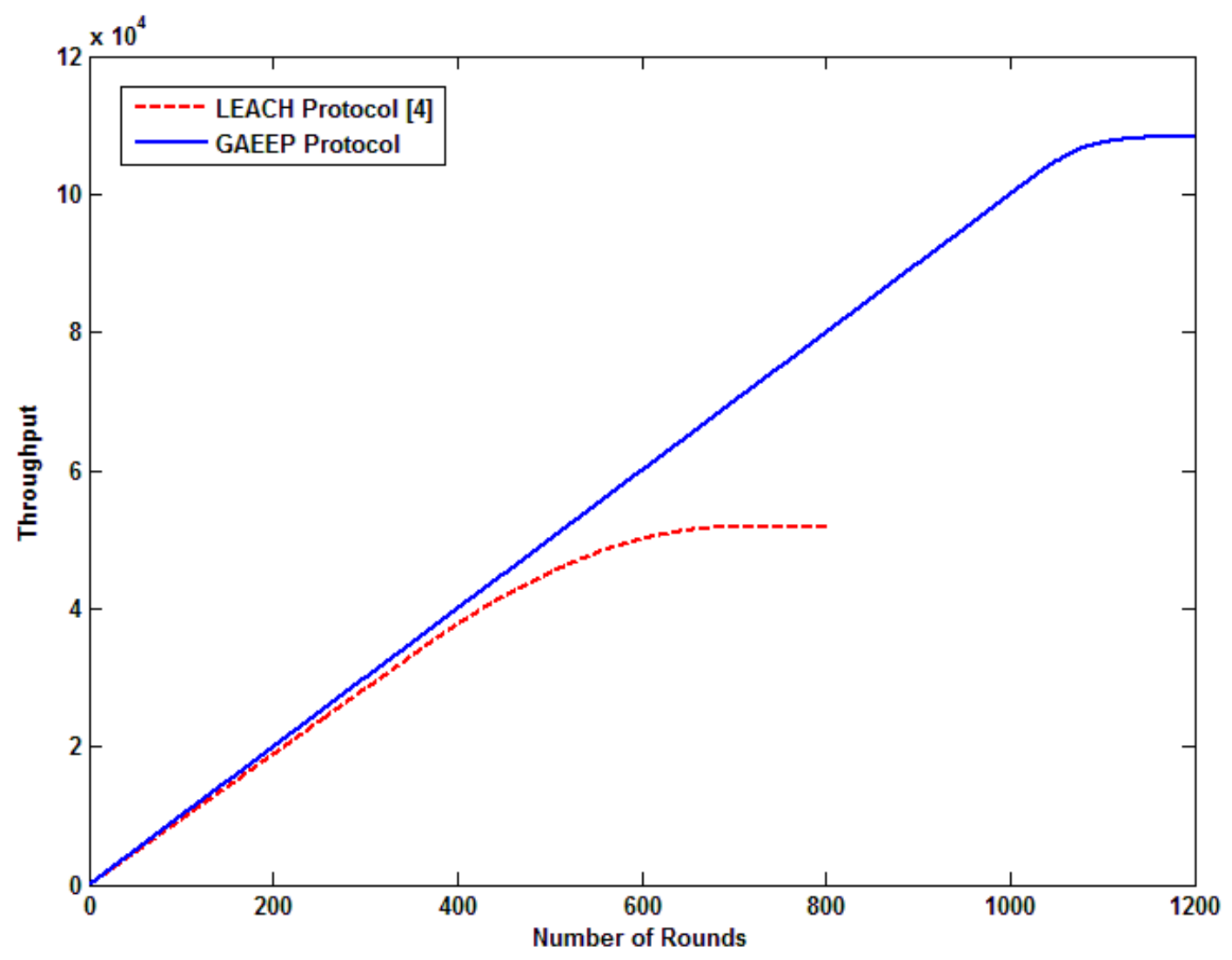

Figure 10. Network Throughput (Experiment 1) 


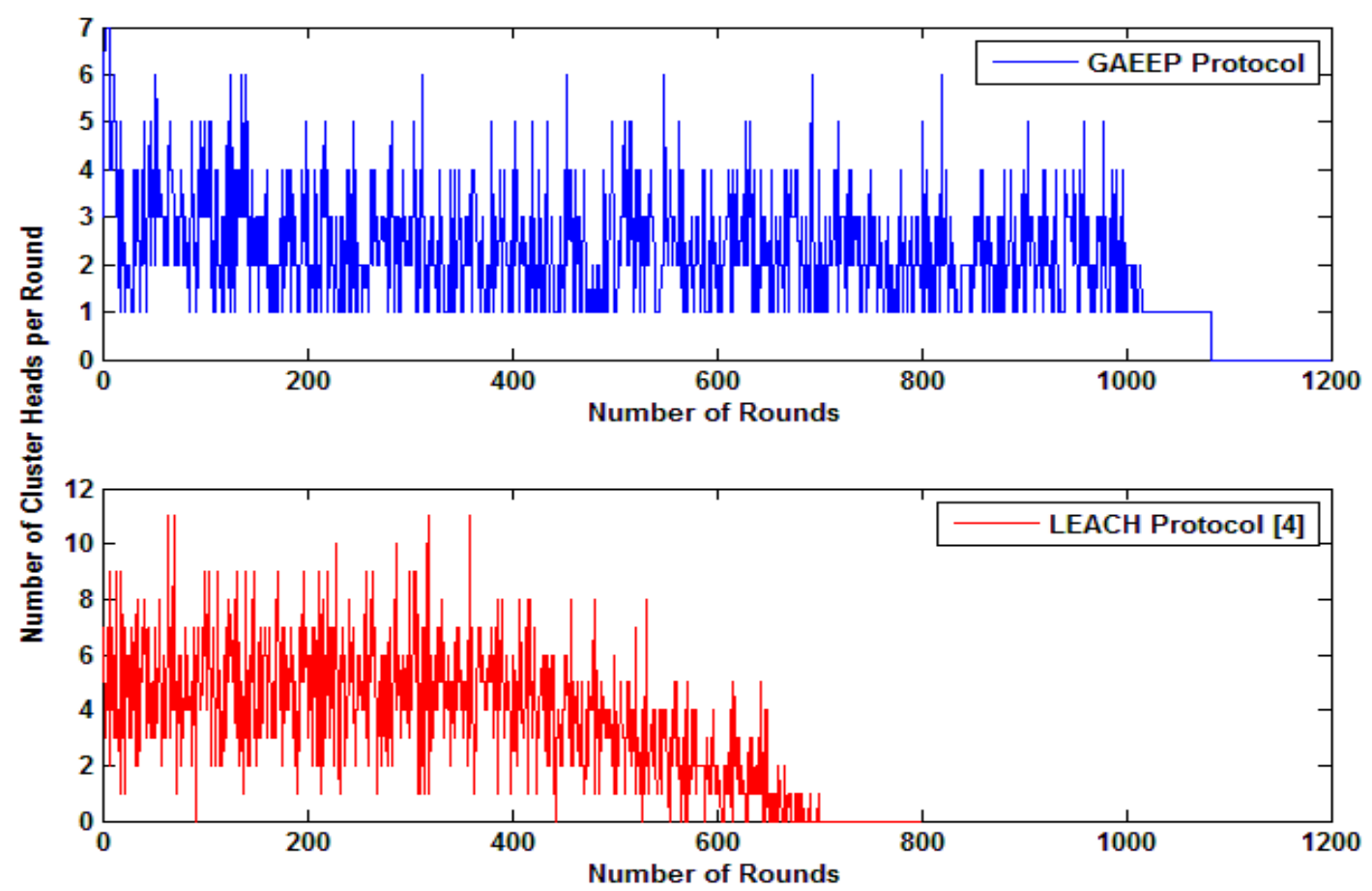

Figure 11. Number of Cluster Head per Round (Experiment 1)

\subsubsection{Experiment 2}

Let us assume the heterogeneous case where a percentage of the population of sensors nodes is equipped with more energy resources than the rest of the nodes. Let $m$ be the fraction of the total number of nodes $N$, which are equipped with $\alpha$ times more energy than the others. These powerful nodes are called advanced nodes, and the rest $(1-m) \times N$ are called normal nodes. To study the effect of heterogeneity on the performance of the proposed GAEEP protocol and previous protocols such as LEACH [4], SEP [15] and ERP [16], we consider the same simulation parameters as in [4, 15-16]. The simulation consists of 90 nodes with initial energy of $0.5 \mathrm{~J}$ plus 10 advanced nodes with initial energy of $1 \mathrm{~J}$ (i.e., $m=0.1$ and $\alpha=1$ ), scattered randomly within a $100 \times 100 \mathrm{~m}^{2}$ sensor field as shown in Figure (12) and the packets sent were 4000 bits plus 50 bits as control packet. BS is placed at the center of the sensor field. The GA parameters are set as experiment 1.

The network lifetime for the proposed GAEEP protocol and other protocols is shown in Figure 13. Figure 14 shows the number of rounds at FDN, HDN and LDN for LEACH [4], SEP [15], ERP [16] and GAEEP protocols. From these figures, it can be observed that the proposed GAEEP protocol in both homogeneous and heterogeneous cases outperforms the other protocols in terms of stability period. The heterogeneous GAEEP improves the network lifetime by 2726 rounds as compared to homogenous GAEEP but have slightly effect on the stability period. This means that the advanced nodes stay live for long time because they have more energy than normal nodes and this increase the network lifetime.

The heterogeneous GAEEP protocol extends the stability period by 386.1, 330.9 and 304.6 rounds as compared to LEACH, SEP and ERP respectively. Moreover, GAEEP improves the network lifetime by $110.53 \%, 139.6 \%$ and $48.22 \%$ over LEACH, SEP and ERP protocols respectively. Figure (15) shows that the residual energy of all nodes obtained using heterogeneous GAEEP protocol decreases more slowly than other protocols. After 1460 rounds, the sensor nodes have $3.487 \mathrm{~J}, 0.7527 \mathrm{~J}$ and $5.928 \mathrm{~J}$ in LEACH, SEP and ERP protocols, respectively. However, the sensor nodes in heterogeneous GAEEP protocol have $13.25 \mathrm{~J}$. Finally, we conclude that the GAEEP is 
energy efficient protocol and improves the stability period for homogeneous and heterogeneous cases.

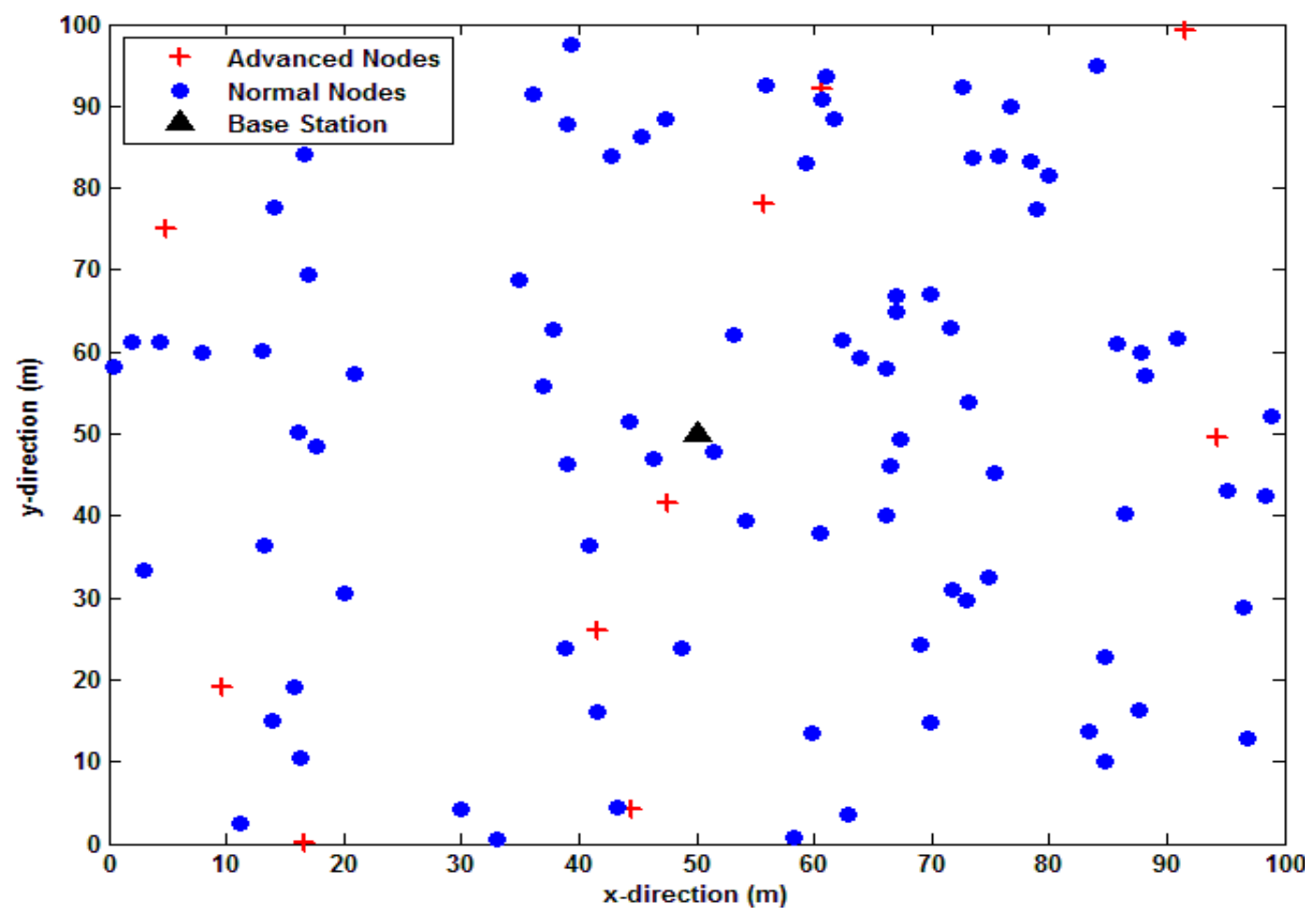

Figure 12. Heterogeneous Network before Clustering (Experiment 2)

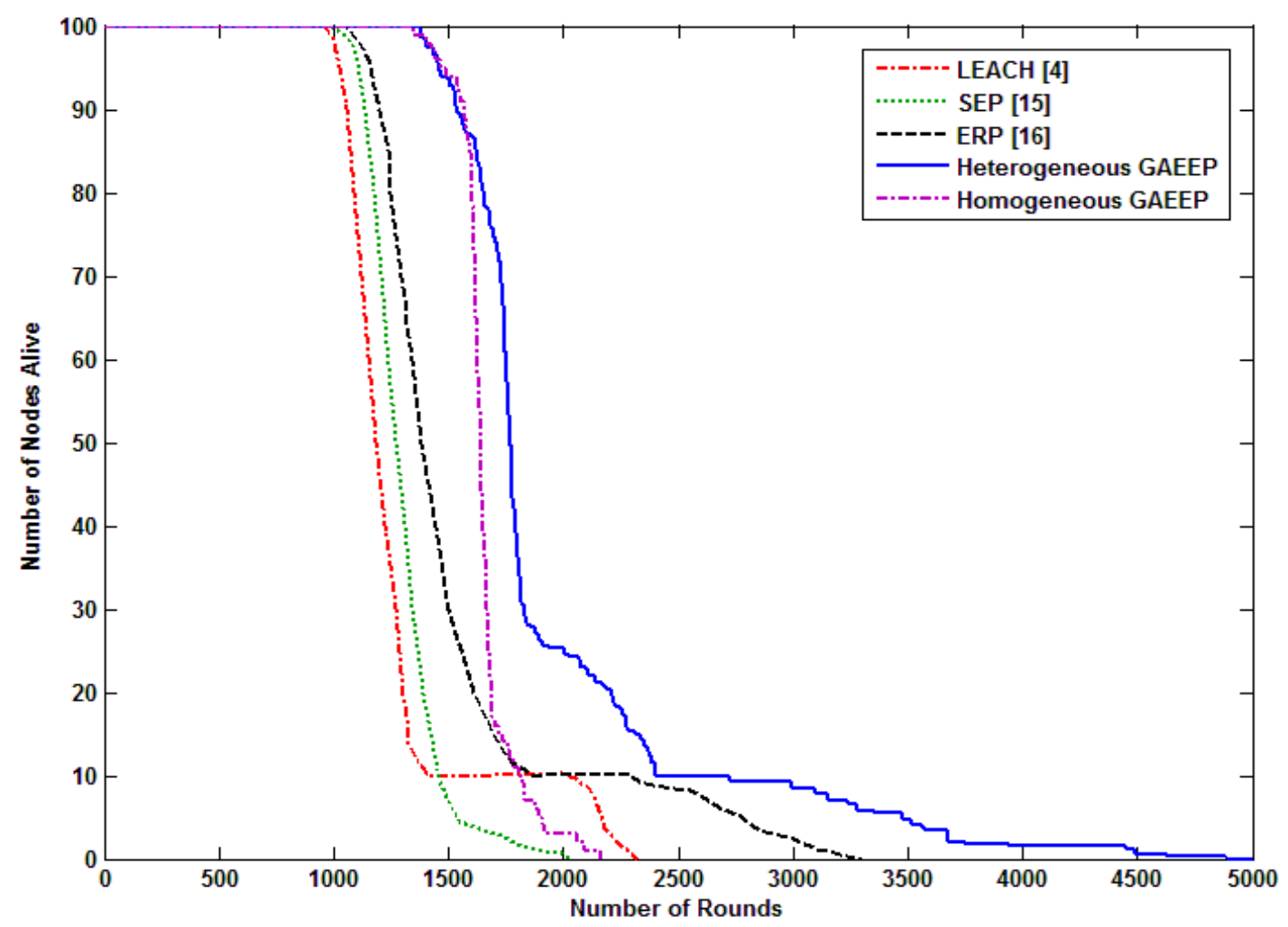

Figure 13. Network Lifetime Obtained using Different Protocols (Experiment 2) 


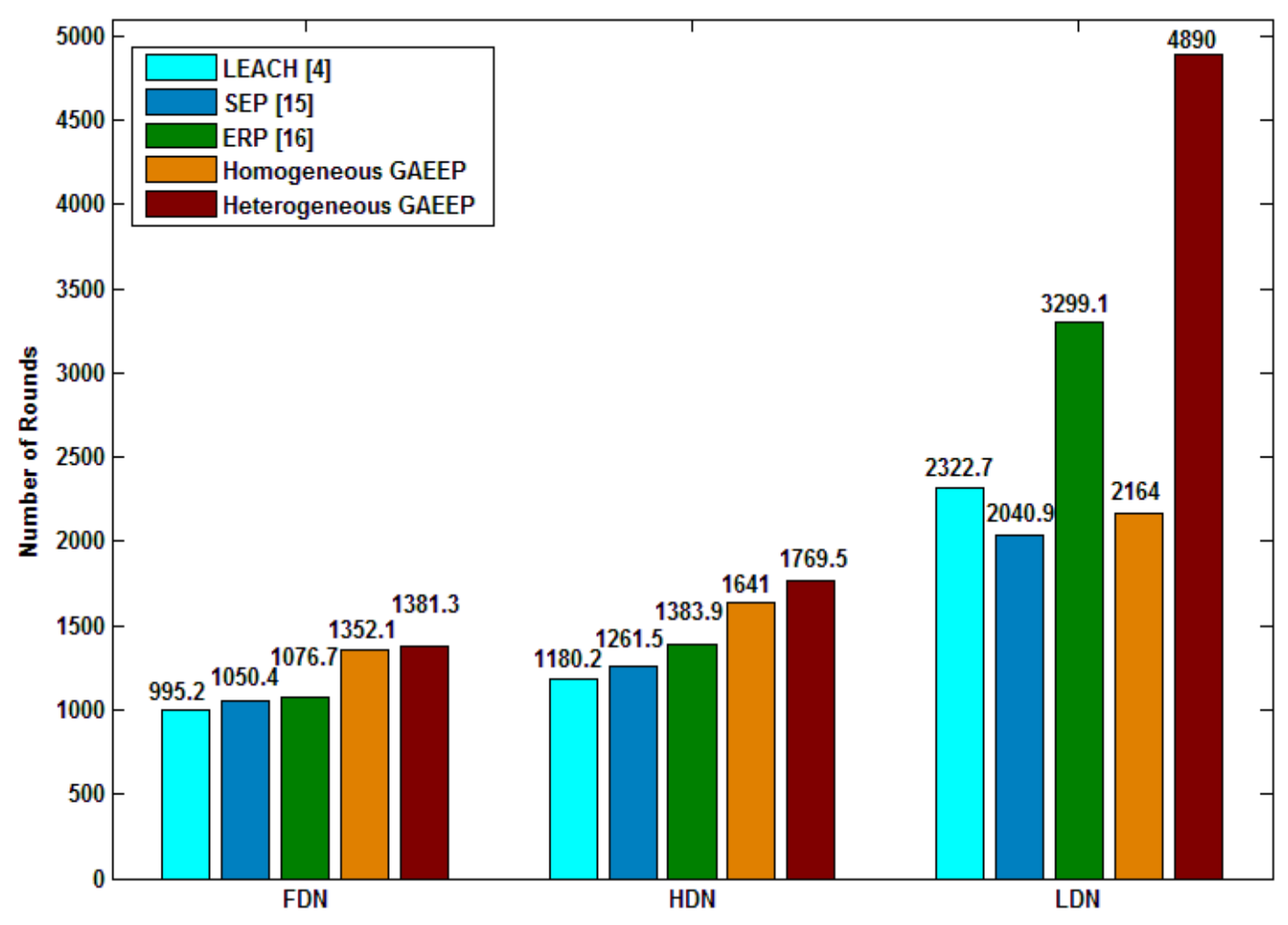

Figure 14. Number of Rounds at FDN, HDN and LDN (Experiment 2)

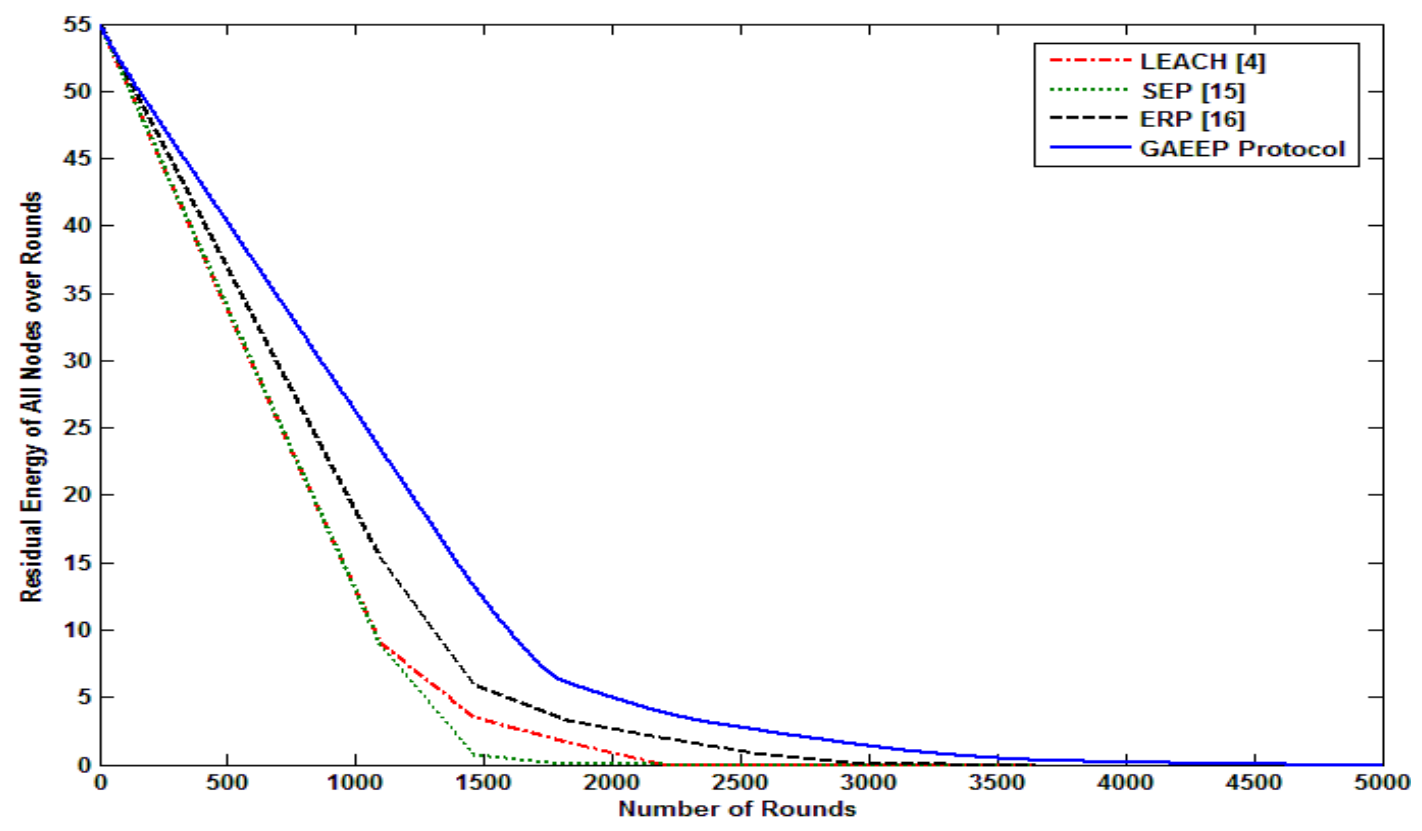

Figure 15. Residual Energy of Heterogeneous Network (Experiment 2)

\subsubsection{Experiment 3}

In this experiment, we study the effect of BS position on the performance of the proposed protocol and compare its performance with LEACH [4], LEACH-GA [8] and ALEACH [9] protocols. The simulation consists of 100 homogeneous nodes with initial energy of $0.5 \mathrm{~J}$, scattered randomly within a $50 \times 50 \mathrm{~m}^{2}$ sensor field. The packets sent were 2000 bits plus 50 bits control packets same as the settings in [8] and [9]. The GA parameters are set as $p_{s}=30, p_{m}=0.1, p_{c}=0.7, w=0.9$ and Maxgen $=150$. 
Figures 16-19 show the number of rounds required when the number of dead nodes is $1 \%(\mathrm{FDN}), 20 \%, 50 \%(\mathrm{HDN})$ and 100\% (LDN) respectively for LEACH [4], LEACHGA [8], A-LEACH [9] and GAEEP protocols at different vertical positions (y) of BS; namely at $(25 \mathrm{~m}, 25 \mathrm{~m}),(25 \mathrm{~m}, 100 \mathrm{~m})$ and $(25 \mathrm{~m}, 250 \mathrm{~m})$. From these figures, it is noticed that the number of rounds is large when the BS at the center of sensor field $(25 \mathrm{~m}$, $25 \mathrm{~m}$ ) and it decreases when the BS moves outward. Also, it is obvious that the stable period of the proposed GAEEP protocol extended as compared to LEACH, LEACH-GA and A-LEACH by $1834.7,1720.7$ and 1515.7 rounds respectively when BS at $(25 \mathrm{~m}, 25$ $\mathrm{m})$ and by $1009.5,711.5$ and 202.5 rounds respectively when $\mathrm{BS}$ at $(25 \mathrm{~m}, 250 \mathrm{~m})$. It is observed that the stable period obtained using GAEEP protocol when the BS at center of the sensor field is wider than that obtained when BS moves outward. This due to that the free space energy model is used when the BS located at $(25 \mathrm{~m}, 25 \mathrm{~m})$ because the distances between BS and the sensor nodes always less than the threshold distance $\left(d_{o}\right)$.

This decreases the dissipated energy and extends the stable period. However, the distances between BS and the sensor nodes increase when the BS moves outward and this increases the dissipated energy. Moreover, the instability period is narrow as compared other protocols and this increases the reliability of GAEEP protocol in the clustering process of WSN.

From Figure 19, it is cleared that the proposed protocol improves the network lifetime by $105.67 \%, 86.52 \%$ and $50.04 \%$ as compared to LEACH, LEACH-GA and A-LEACH respectively when BS located the center of the sensor field. When the BS located at (25 $\mathrm{m}, 100 \mathrm{~m}$ ), the network lifetime improved by $75.88 \%, 73.17 \%$ and $42.28 \%$ as compared to three LEACH protocols. Finally, the network lifetime improved by $131.89 \%, 50.54 \%$ and $7.49 \%$ as compared to LEACH, LEACH-GA and A-LEACH respectively when BS located at $(25 \mathrm{~m}, 250 \mathrm{~m})$. The network lifetime decreases as the BS moves far away from the sensor field as shown in Figure 20. Figure 21 shows that the residual energy of all nodes obtained using GAEEP protocol decreases more slowly, when BS at $(25 \mathrm{~m}, 25 \mathrm{~m})$, than other cases when BS moves far away from the center of the sensor field.

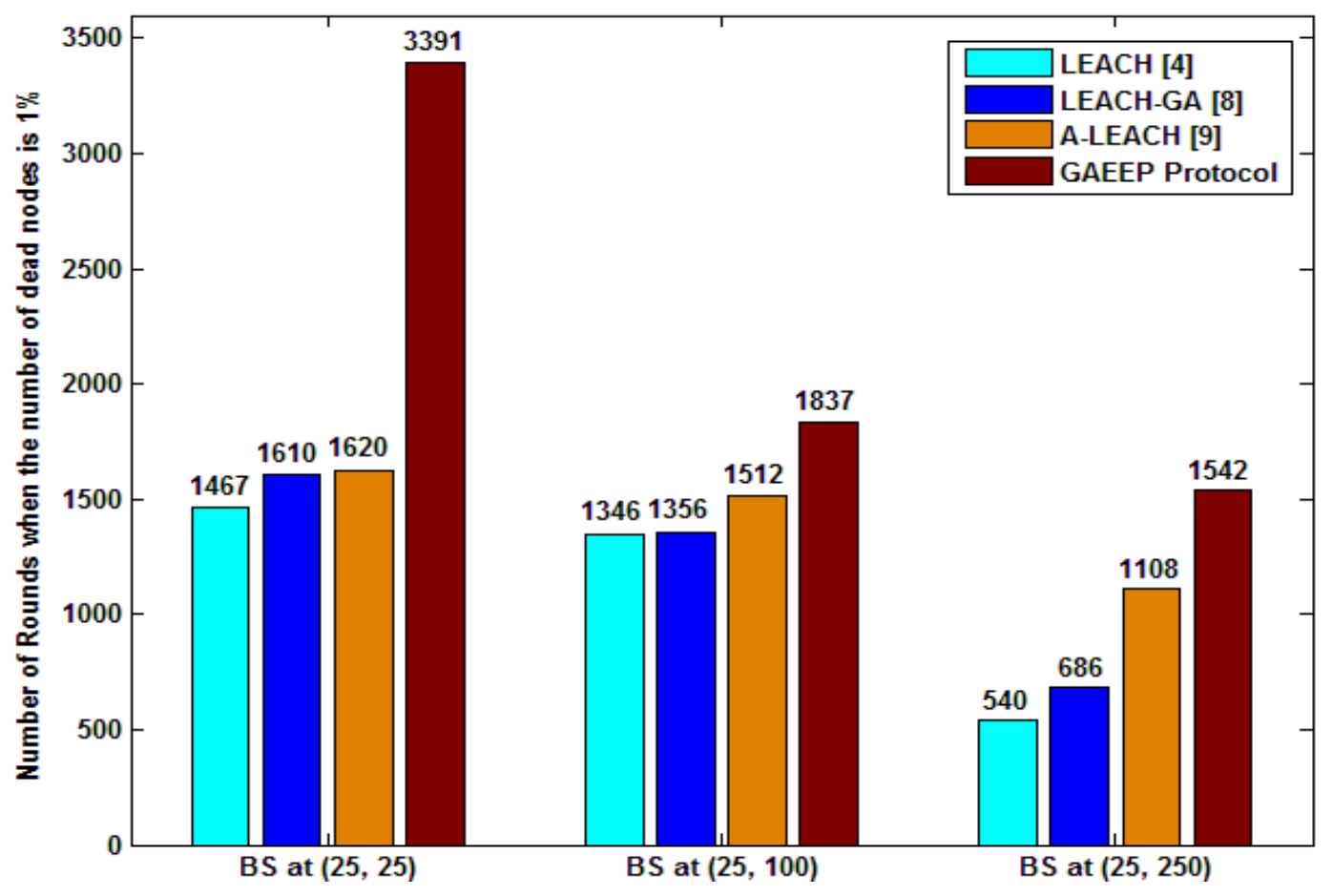

Figure 16. Number of Rounds obtained when the Number of Dead Nodes is 1\% (Experiment 3) 


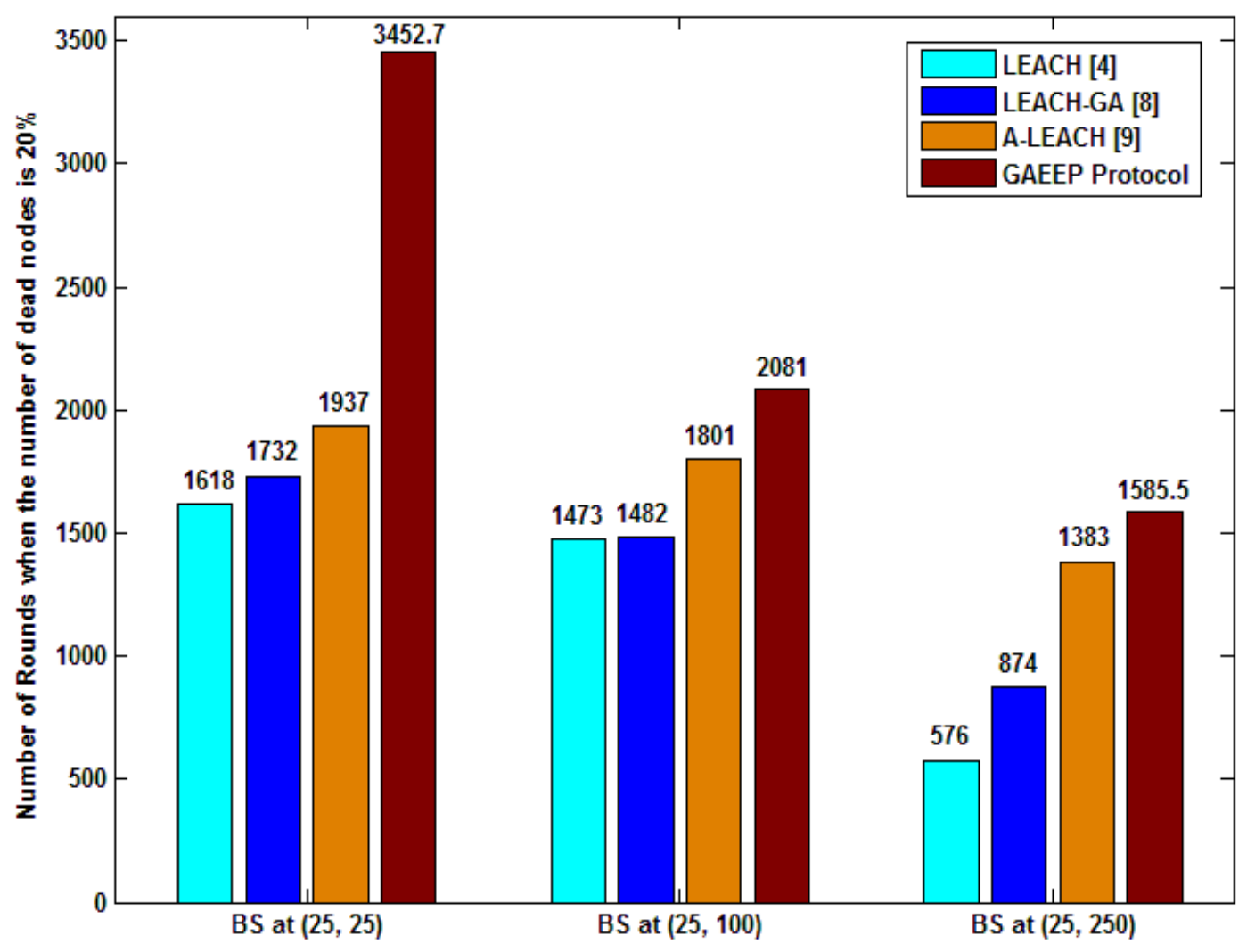

Figure 17. Number of Rounds Obtained when the Number of Dead Nodes is 20\% (Experiment 3)

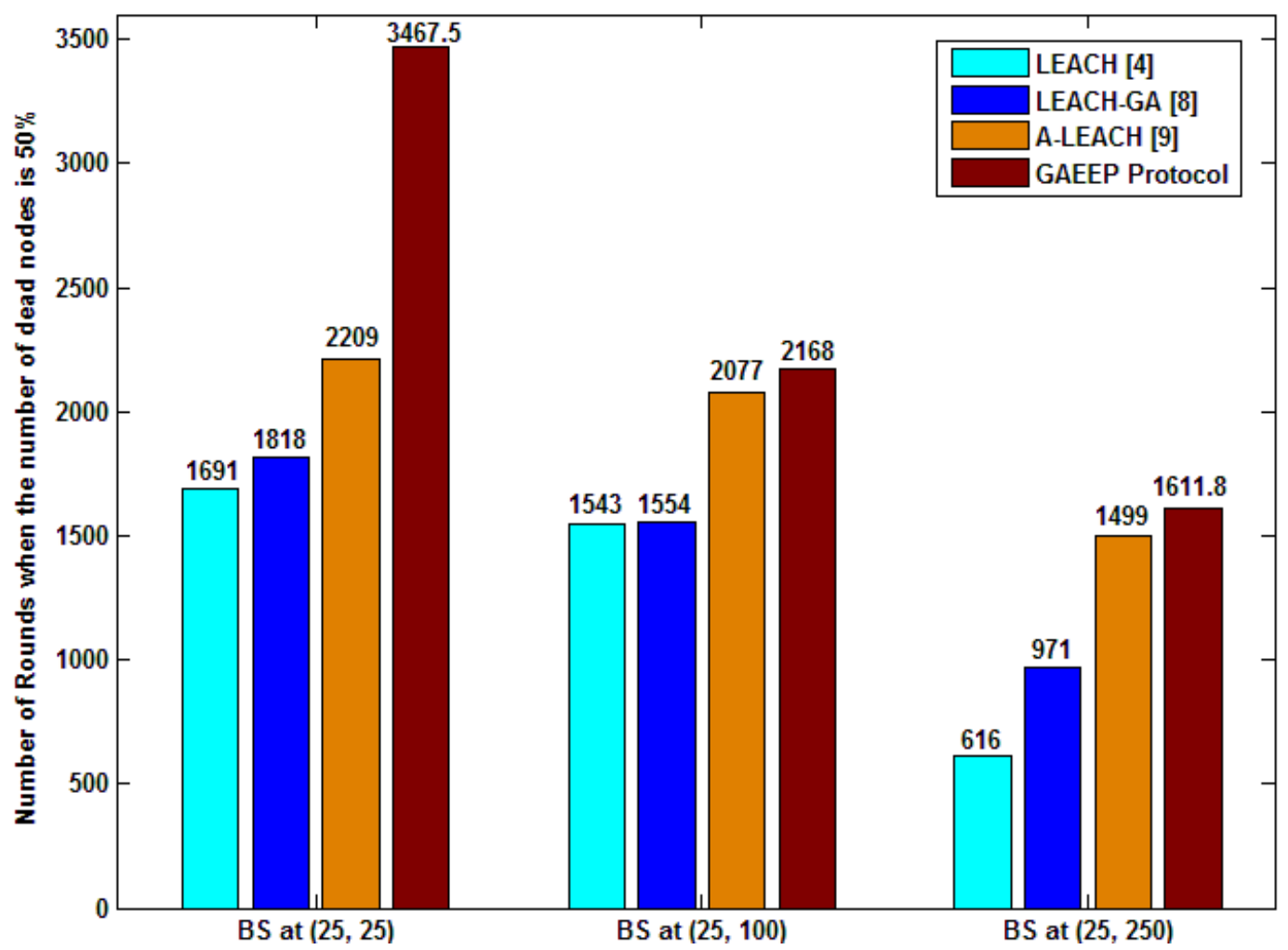

Figure 18. Number of Rounds obtained when the Number of Dead Nodes is $50 \%$ (Experiment 3) 


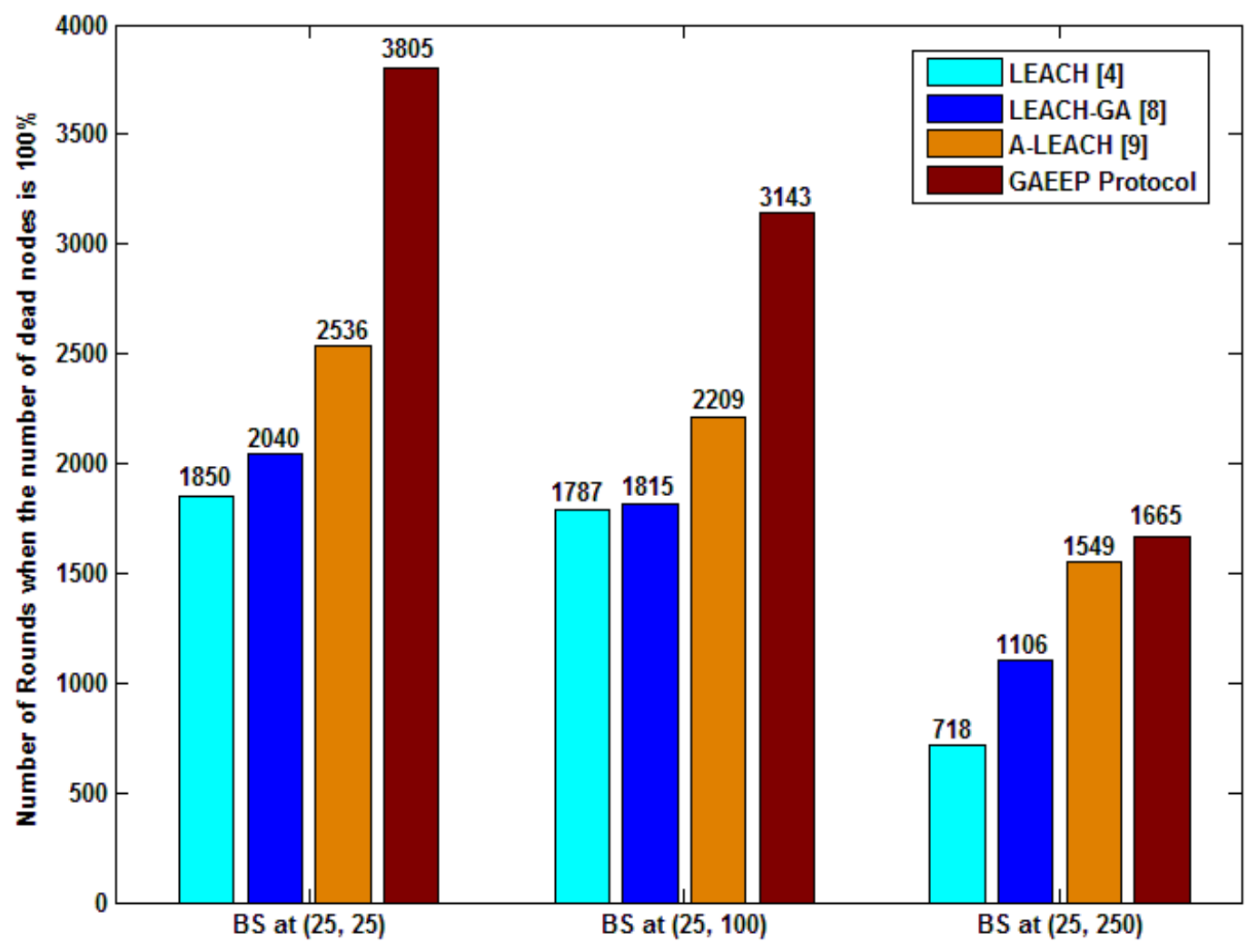

Figure 19. Number of Rounds Obtained when the Number of Dead Nodes is $100 \%$ (Experiment 3)

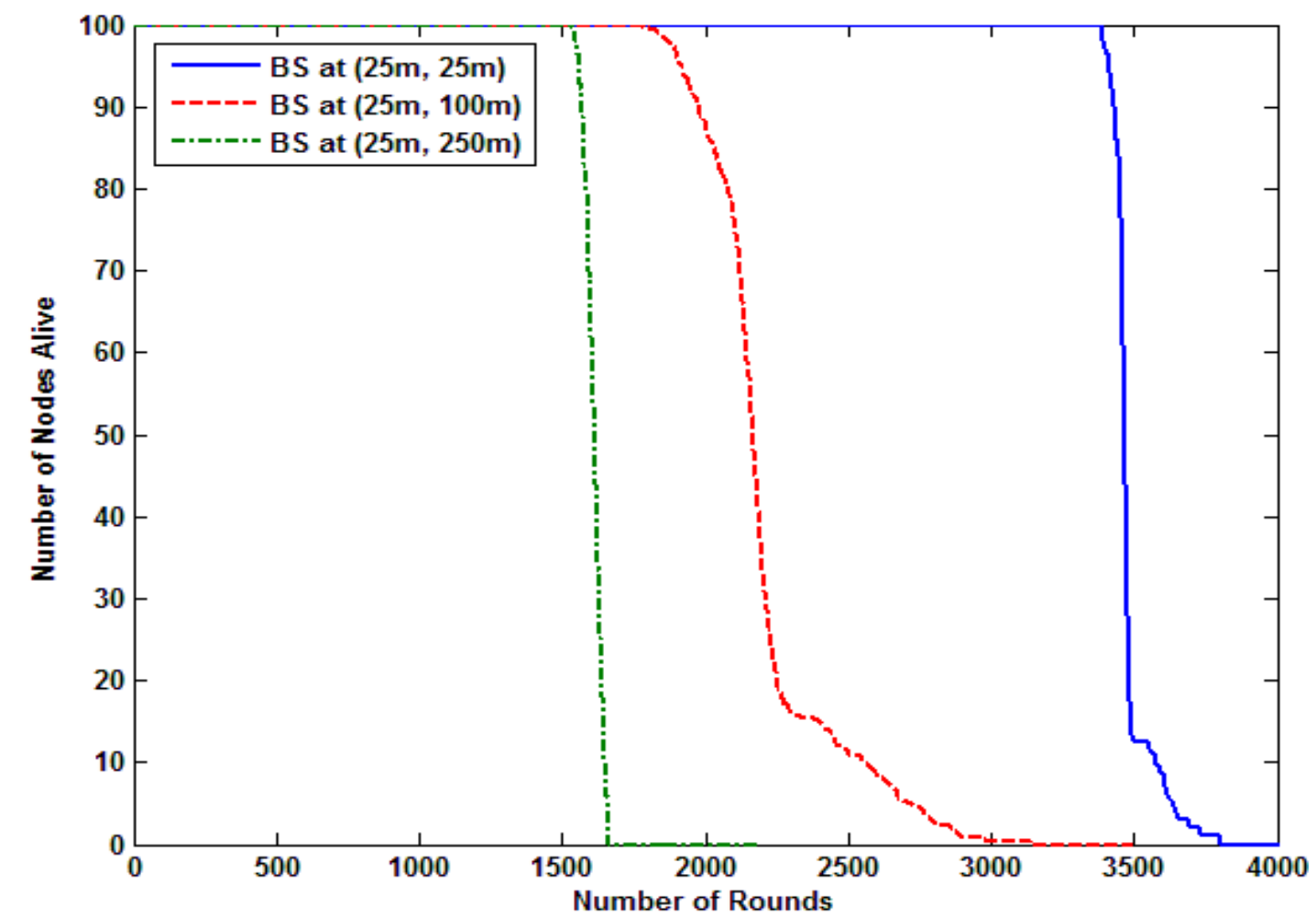

Figure 20. Network Lifetime Obtained using GAEEP Protocol at Different BS Locations (Experiment 3) 


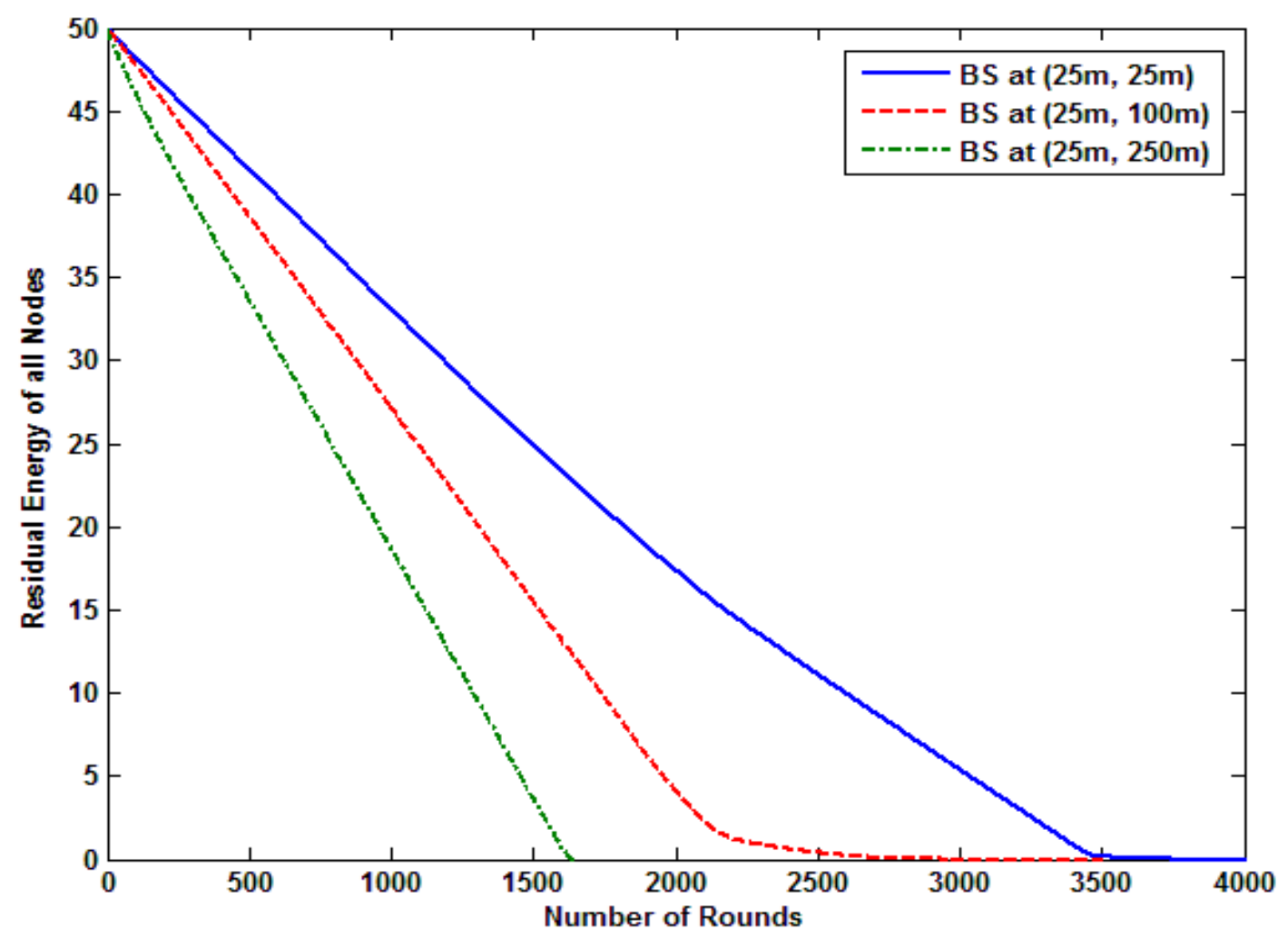

Figure 21. Residual Energy of all Nodes Obtained using GAEEP Protocol at Different BS Locations (Experiment 3)

\subsubsection{Experiment 4}

Finally, this experiment studies the performance of the proposed protocol in high dense sensors network and compares it with the DEU protocol that described in [17]. The simulation considers 400 sensor nodes with initial energy of $0.5 \mathrm{~J}$, scattered randomly within a $200 \times 200 \mathrm{~m}^{2}$ sensor field and the packets sent were 4000 bit same as the settings in [17] plus 50 bits control packet. The BS location is at center of sensor field $(100,100)$ $\mathrm{m}$. The GA parameters are set as $\mathrm{p}_{\mathrm{s}}=30, \mathrm{p}_{\mathrm{m}}=0.04, \mathrm{p}_{\mathrm{c}}=0.7, w=0.9$ and Maxgen $=150$.

Figure 22 shows the network after clustering using GAEEP protocol at certain round. The networks lifetime obtained using DEU and GAEEP protocols are shown in Figure 23. From this figure, it is obvious that, the first node in DEU protocol died after 750 rounds, and all nodes died after 800 rounds. However in GAEEP protocol, the first node died after 1055.5 rounds, and all nodes died after 1978 rounds. This means that the stable region of the proposed protocol extended as compared to the DEU by 305.5 rounds. Moreover, the proposed GAEEP protocol improves the network lifetime by $147.25 \%$ over DEU protocol. The total residual energy of all sensor nodes in the network for GAEEP and DEU protocols is shown in Figure 24. From this figure, it is noticed that the residual energy for GAEEP protocol decreases more slowly than that for DEU protocol. In DEU protocol, the total residual energy dissipates after 800 rounds. However in GAEEP protocol, the total residual energy dissipates after 1978 rounds.

Figure 25 shows the variance of nodes' residual energy along rounds for GAEEP and DEU protocols. It is cleared the residual energy's variance of the nodes in GAEEP is lowest and has smallest variation (approximately $0.24 \times 10^{-3}$ ) as the residual energy's variance of the nodes in DEU (approximately $0.2 \times 10^{-3}$ ). This means that GAEEP protocol can effectively balance the energy consumption in the network. Moreover, it saves the energy of single node and prolongs the lifetime of the network. 


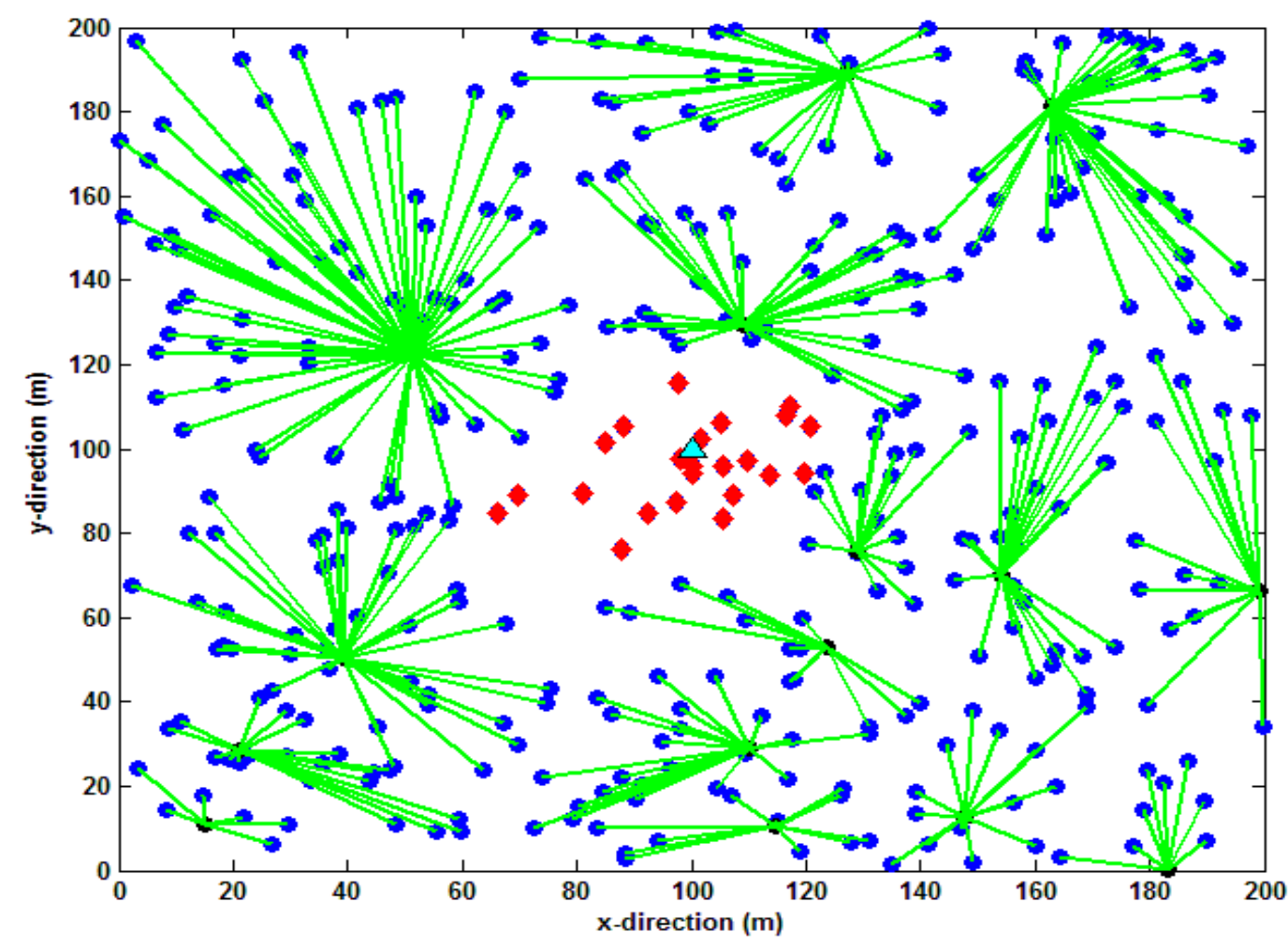

Figure 22. Network after Clustering (Experiment 4); where $\Delta$ is BS and ‘'s are Sensor Nodes that are Connected to BS Directly

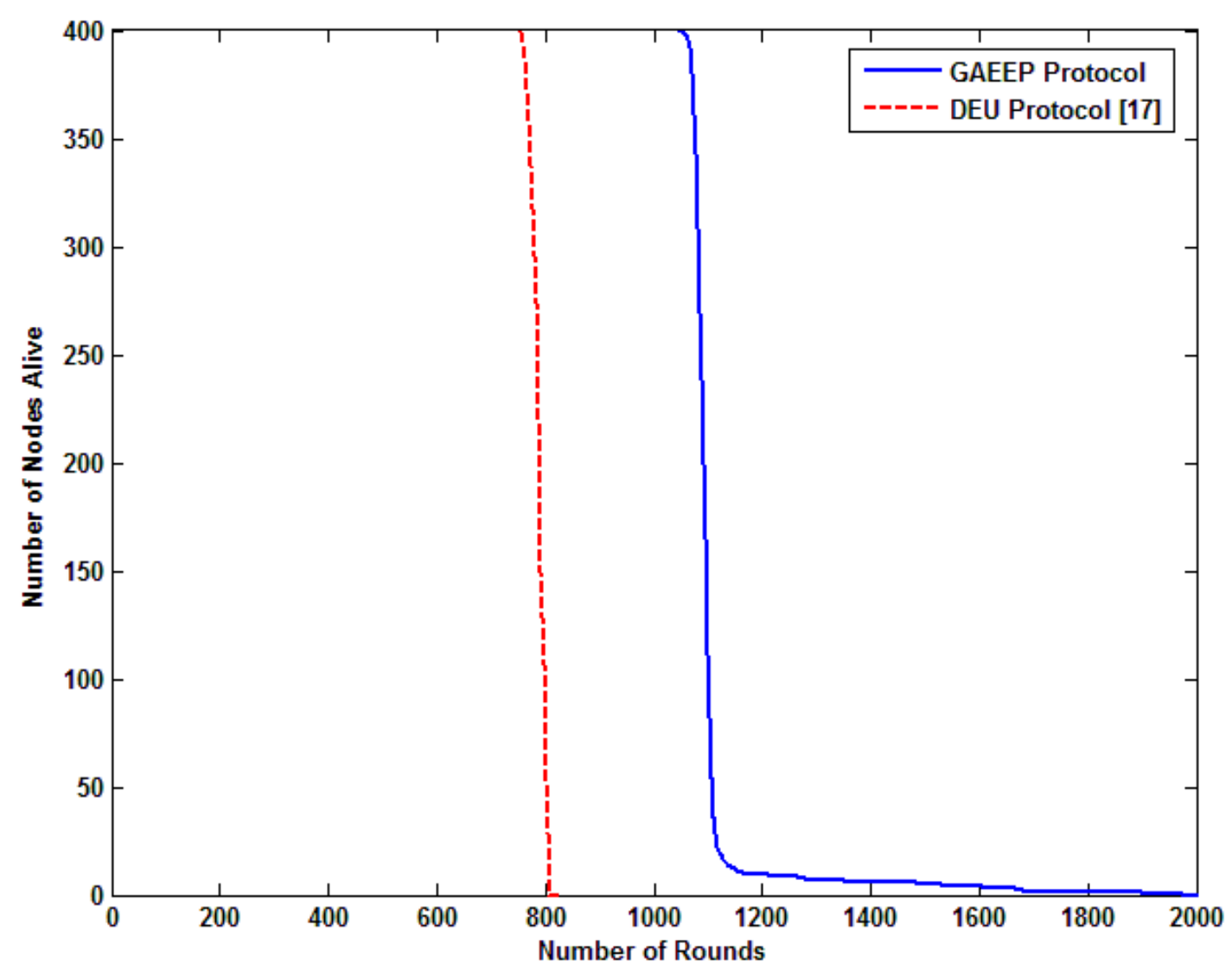

Figure 23. Network Lifetime Obtained using DEU and GAEEP Protocols (Experiment 4) 


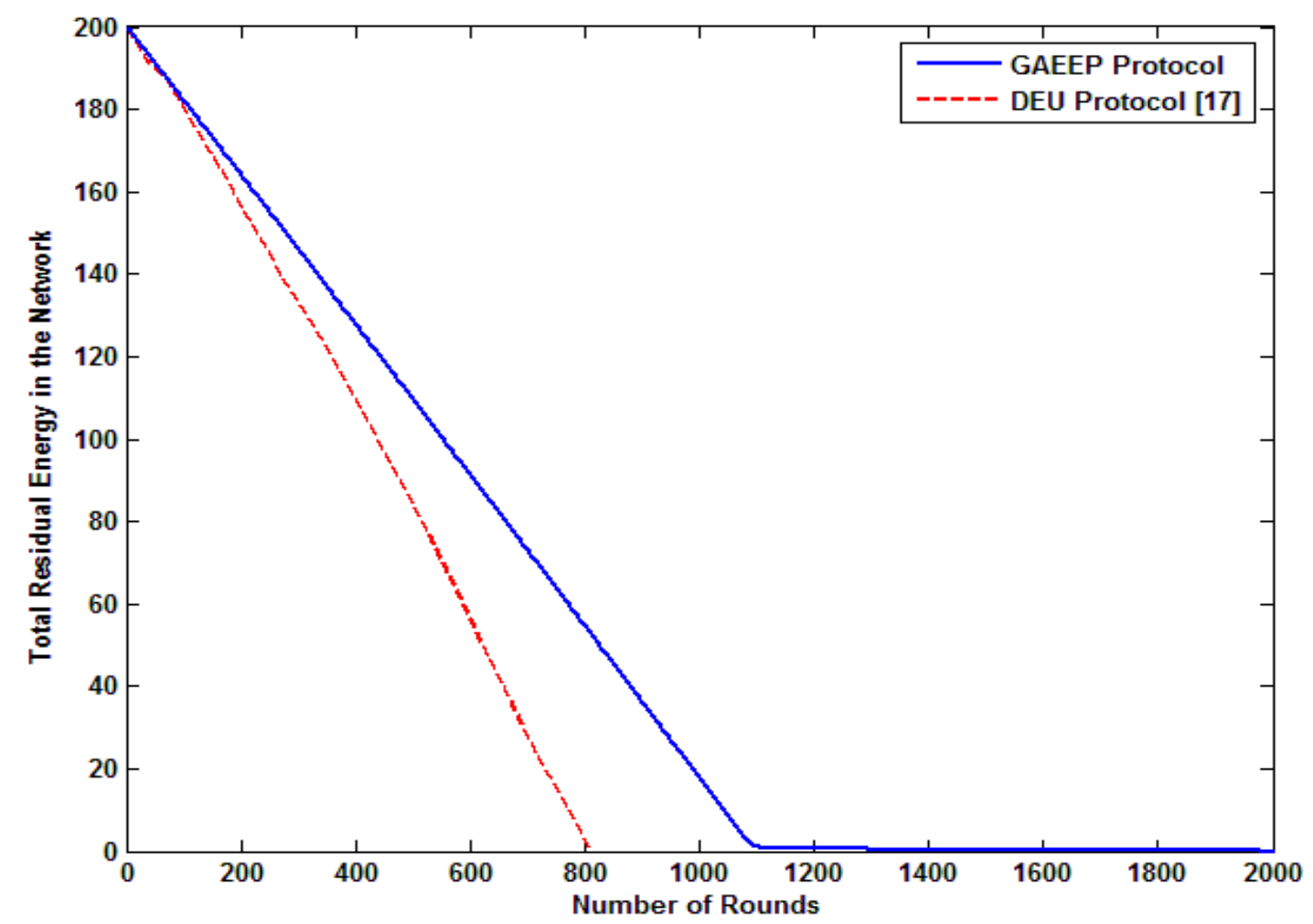

Figure 24. Total Residual Energy of all Sensor Nodes for DEU and GAEEP Protocols (Experiment 4)

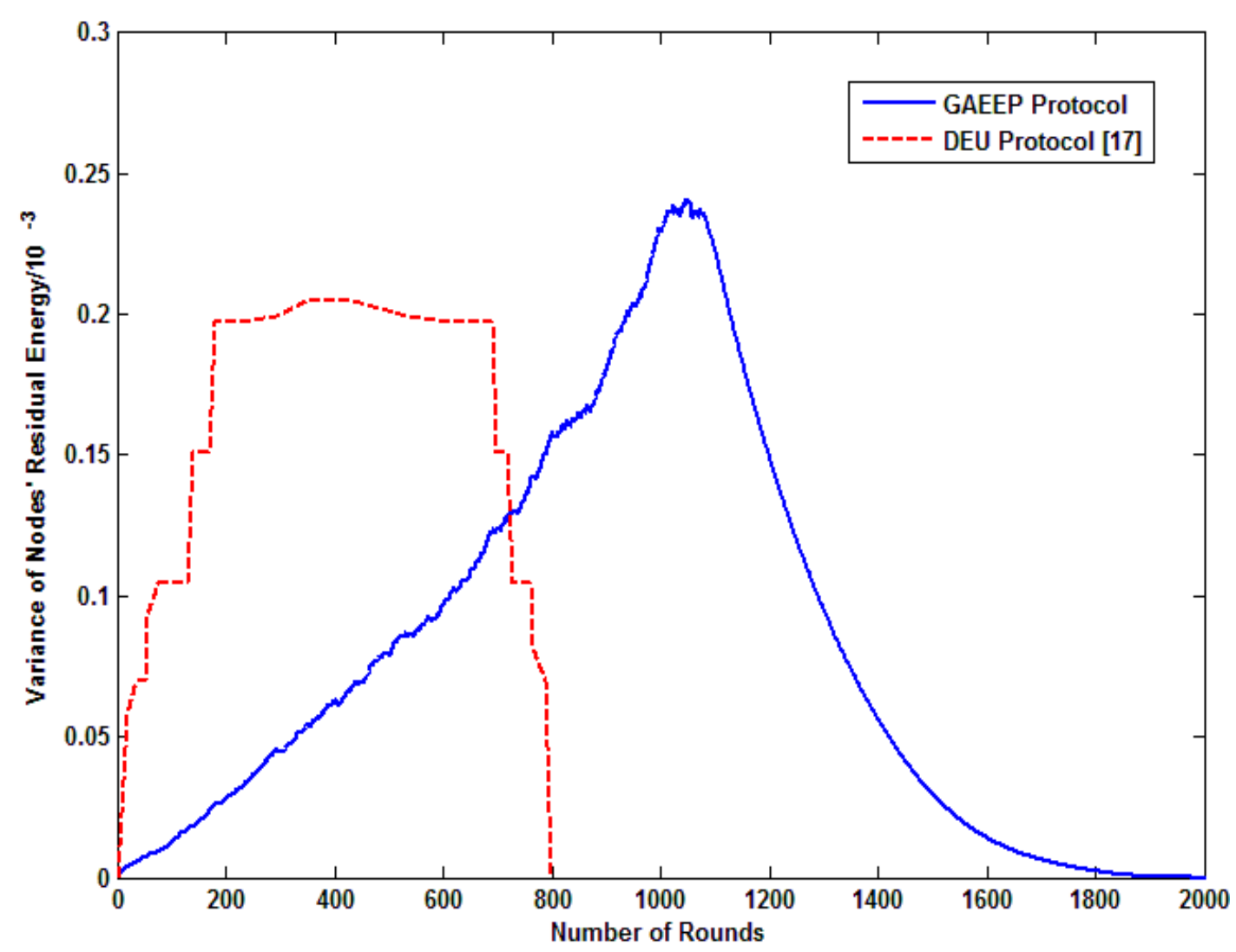

Figure 25. Variance of Nodes' Residual Energy along Rounds (Experiment 


\section{Conclusion}

In this paper, a new Genetic Algorithm-based Energy-Efficient adaptive clustering hierarchy Protocol (GAEEP) has been presented to efficiently maximize the lifetime and stability period of wireless sensor networks. GAEEP uses genetic algorithm to improve the network lifetime and stability period of the wireless sensor networks by finding the optimum number of cluster heads and their locations based on minimizing the energy consumption of the sensor nodes. Matlab simulation results showed that the proposed GAEEP protocol is more energy efficient and more reliable in clustering process as compared to LEACH, SEP, ERP, LEACH-GA, A-LEACH and DEU protocols in low or high dense networks and in homogeneous or heterogeneous networks. Also, GAEEP protocol increases the reliability of clustering process because it expands the stability period and compresses the instability period. Moreover, it outperforms the previous protocols in terms of energy dissipation rate, network lifetime and stability period in both homogeneous and heterogeneous cases.

\section{References}

[1] S. K. Singh, M. P. Singh and D. K. Singh, "Routing Protocols in Wireless Sensor Networks - A Survey", Int. Journal of Computer Science and Engineering Survey (IJCSES), vol. 1, no. 2, (2010) November, pp. 63-83.

[2] S. A. Sayyadi, R. Senemar and H. Teimuri, "Elect Distance Efficient Cluster Heads for Improvement Performance of LEACH Protocol", $2^{\text {nd }}$ International Conference on Computational Techniques and Artificial Intelligence (ICCTAI'2013), Dubai, (2013) March 17-18, pp. 179-183.

[3] A. A. Abbasi and M. Younis, "A Survey on Clustering Algorithms for Wireless Sensor Networks", Int. Journal of Computer Communications, vol. 30, (2007) June, pp. 2826-2841.

[4] W. Heinzelman, A. Chandrakasan and H. Balakrishnan, "An Application-Specific Protocol Architecture for Wireless Microsensor Networks," IEEE Transactions on Wireless Communications, vol. 1, no. 4, (2002) October, pp. 660-670.

[5] F. Xiangning and S. Yulin, "Improvement on LEACH Protocol of Wireless Sensor Network", Conference on Sensor Technologies and Applications, Valencia, (2007) October 14-20, pp. 260-264.

[6] V. Loscrì, G. Morabito and S. Marano, "A Two-Level Hierarchy for Low-Energy Adaptive Clustering Hierarchy”, IEEE $62^{\text {nd }}$ Vehicular Technology Conference, (2005) September 25-28, pp. 1809-1813.

[7] M. B. Young, A. Al-zou'bi, Y. Khamayseh and W. Mardini, "Improvement on LEACH Protocol of Wireless Sensor Network (VLEACH)", International Journal of Digital Content Technology and Its Applications, vol. 3, no. 2, (2009), pp. 132-136.

[8] J. L. Liu and C. V. Ravishankar, "LEACH-GA: Genetic Algorithm-Based Energy-Efficient Adaptive Clustering Protocol for Wireless Sensor Networks", Int. J. of Machine Learning and Computing, vol. 1, no. 1, (2011) April, pp. 79-85.

[9] K. Go. Vijayvargiya and V. Shrivastava, "An Amend Implementation on LEACH protocol based on Energy Hierarchy", International Journal of Current Engineering and Technology, vol. 2, no. 4, (2012) December, pp. 427-431.

[10] R. Halke and V. A. Kulkarni, "En-LEACH Routing Protocol for Wireless Sensor Network", International Journal of Engineering Research and Applications (IJERA), vol. 2, no. 4, (2012) August, pp. 2099-2102.

[11] J. S. Brunda, B. S. Manjunath, B. R. Savitha and P. Ullas, "Energy Aware Threshold based Efficient Clustering (EATEC) for Wireless Sensor Network", International Journal of Computer Technology and Electronics Engineering (IJCTEE), vol. 2, no. 4, (2012) August, pp. 25-30.

[12] W. Liu and L. Wang, "An improved algorithm based on LEACH protocol", Journal of Applied Mechanics and Materials, vol. 347, (2013), pp. 2725-2727.

[13] K. Pawar, V. Pawar and T. Sharma, "Enhancement of LEACH Protocol Using Energy Heterogeneity Concept", International Journal of Emerging Trends and Technology in Computer Science (IJETTCS), vol. 2, no. 1, (2013) February, pp. 49-56.

[14] P. Bakaraniya and S. Mehta, "K-LEACH: An improved LEACH Protocol for Lifetime Improvement in WSN", International Journal of Engineering Trends and Technology (IJETT), vol. 4, no. 5, (2013) May, pp. 1521-1526.

[15] G. Smaragdakis, I. Matta and A. Bestavros, "SEP: A Stable Election Protocol for clustered heterogeneous wireless sensor networks", Second International Workshop on Sensor and Actor Network Protocols and Applications (SANPA), (2004).

[16] B, A. Attea and E. A. Khalil, "A New Evolutionary Based Routing Protocol for Clustered Heterogeneous Wireless Sensor Networks", Applied Soft Computing, vol. 12, no. 7, (2012) July, pp. $1950-1957$. 
[17] C. Zhang, F. Liu and N. Wu, "A Distributed Energy-efficient Unequal Clustering Routing Protocol for Wireless Sensor Networks", Int. Journal of Computational Information Systems, vol. 10, no. 6, (2014) March, pp. 2369-2376.

[18] M. Wu, "Energy- Efficient Routing Protocols in Heterogeneous Wireless Sensor Networks", Ph. D. thesis, Faculty of Engineering \& Computing, Dublin City University, (2012) October.

[19] T. D. Singh," Analysis of Low Energy Adaptive Clustering Hierarchy (LEACH) protocol”, National Institute of Technology Rourkela, India, (2011).

[20] M. Gen and R. Cheng, "Genetic Algorithm and Engineering Optimization", John Wiley and Sons, New York, (2000).

[21] J. T. Tsai, J. H. Chou and T. K Liu, "Optimal Design of Digital IIR Filters by Using Hybrid Taguchi Genetic Algorithm”, IEEE Trans. on Industrial Electronics, vol. 53, no. 3, 2, (2006), pp. 867-879.

[22] S.-M. Im and J-J. Lee, "Adaptive Crossover, Mutation and Selection using Fuzzy System for Genetic Algorithms", Artificial Life and Robotics, vol. 13, no. 1, (2008), pp. 129-133.

\section{Authors}

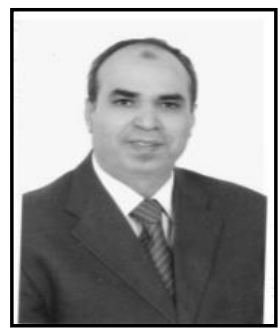

Prof. Mohammed Abo-Zahhad, (SIEEEM'00) received his B.S.E.E. and M.S.E.E degrees in electrical engineering in 1979 and 1983 respectively, both from Assiut University, Egypt . In 1988, he received Ph. D. degree from the University of Kent at Canterbury, UK and Assiut University (channel system). His research interests include switched-capacitor, optical and digital filters, biomedical and genomic signal processing, speech processing, data compression, wavelet-transforms, genetic algorithms, immune algorithms, wireless sensor networks and electronic systems. He has published more than 106 papers in national and international journals and conferences in the above fields. Professor Abo-Zahhad is currently a Professor of Electronics and Communi-cation Engineering, since Jan.1999. Also, he is the director of AU Management Information System (MIS) center and a vice-dean for graduated studies, Faculty of Engineering, Assiut University, since August 2006. He is a member of the European Society of Circuit Theory and Applications, 1998 and a senior IEEE member, 2000.

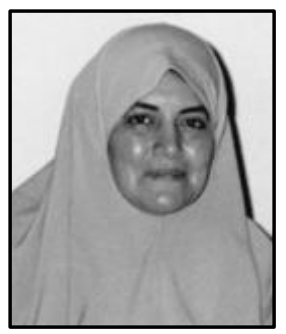

Prof. Sabah M. Ahmed, received her B.S.E.E. and M.S.E.E degrees in electrical engineering in 1979 (excellent with honors) and 1983 respectively, both from Assiut University , Egypt. In 1992, she received $\mathrm{Ph}$. D. degree from the Technical University of Budapest, Hungary. Her research interests include speech processing, biomedical and genomic signal processing, data compression, wavelet-transforms, genetic algorithms, and immune algorithms. She has published more than 56 papers in national and international journals and conferences in the above fields. Professor Sabah is currently a Professor of Electronics and Communication Engineering, since Feb. 2009. Also, she is the director of Faculty of Engineering ICDL center, Assiut University and the manager of Assiut University communication and information technology training center. 


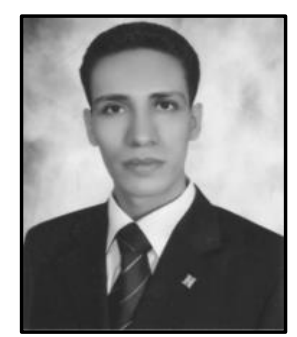

Mr. Nabil Sabor, received his B.S.E.E and M.S.E.E. degrees in electrical engineering in 2006 (excellent with honors) and 2011 respectively, both from Assiut University, Egypt. His research interests include signal and image processing, data compression, biomedical signal processing, wavelet transform, digital filters, genetic algorithms, immune algorithms and wireless sensor networks. He has published more than 6 papers in national and international journals and conferences in the above fields. Mr. Nabil Sabor is currently an Assistant Lecturer in Department of Electronics and Communication Engineering, Assiut University, since May 2011.

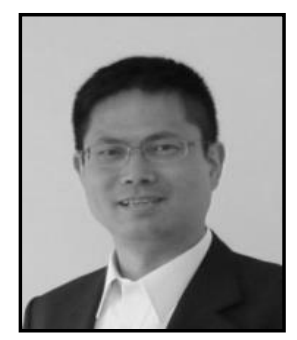

Prof. Shigenobu Sasaki, received B.E., M.E. and Ph.D. degrees from Nagaoka University of Technology, Nagaoka, Japan, in 1987, 1989 and 1993, respectively. Since 1992, he has been with Niigata University, where he is now a Professor at the Department of Electrical and Electronic Engineering. From 1999 to 2000, he was a visiting scholar at the Department of Electrical and Computer Engineering, University of California, San Diego. From 2003 to 2006, he was with the UWB technology institute, National Institute of Information and Communication Technology (NICT) as an expert researcher, where he was involved the R\&D on UWB wireless communications and standardization activities in ITU-R TG1/8. His research interests are in the area of wideband digital communications including spread spectrum systems, ultra-wideband wireless communications and cognitive radio technology. $\mathrm{He}$ is also actively involved in the IEEE 802 standardization on wireless communications over white space, such as the IEEE $802.15 .4 \mathrm{~m}$ and the IEEE 802.22. He is a member of IEEE. 